\title{
Research on Power Flow Transmission through Elastic Structure into a Fluid-Filled Enclosure
}

\author{
Rui Huo $\mathbb{D}^{1,2}$ Chuangye Li $\mathbb{D},^{1,2}$ Laizhao Jing $\mathbb{D},^{1,2}$ and Weike Wang $\mathbb{D}{ }^{1,2}$ \\ ${ }^{1}$ School of Mechanical Engineering, Shandong University, Jinan 250061, China \\ ${ }^{2}$ Key Laboratory of High Efficiency and Clean Mechanical Manufacture (Shandong University), Ministry of Education, \\ Jinan 250061, China \\ Correspondence should be addressed to Rui Huo; huorui@sdu.edu.cn
}

Received 30 October 2017; Revised 8 February 2018; Accepted 15 March 2018; Published 2 May 2018

Academic Editor: Kim M. Liew

Copyright (C) 2018 Rui Huo et al. This is an open access article distributed under the Creative Commons Attribution License, which permits unrestricted use, distribution, and reproduction in any medium, provided the original work is properly cited.

\begin{abstract}
The work of this paper is backgrounded by prediction or evaluation and control of mechanical self-noise in sonar array cavity. The vibratory power flow transmission analysis is applied to reveal the overall vibration level of the fluid-structural coupled system. Through modal coupling analysis on the fluid-structural vibration of the fluid-filled enclosure with elastic boundaries, an efficient computational method is deduced to determine the vibratory power flow generated by exterior excitations on the outside surface of the elastic structure, including the total power flow entering into the fluid-structural coupled system and the net power flow transmitted into the hydroacoustic field. Characteristics of the coupled natural frequencies and modals are investigated by a numerical example of a rectangular water-filled cavity with five acoustic rigid walls and one elastic panel. Influential factors of power flow transmission characteristics are further discussed with the purpose of overall evaluation and reduction of the cavity water sound energy.
\end{abstract}

\section{Introduction}

1.1. Background. The work of this paper is backgrounded by prediction or evaluation and control of mechanical self-noise in sonar array cavity. The mechanical self-noise, which is caused by structural vibration of sonar cavity's wall, might significantly weaken the detection performance of sonar at lower frequencies [1,2]. The sources of mechanical selfnoise might be multiple such as vibrating machines on the ship which diffuse vibration energy or second excitation of structure-borne sound. However, it is essential to comprehend the characteristics of interaction between the enclosed water sound field and its elastic boundary structures for the purpose of prediction, evaluation, and control of interior hydroacoustic noise [3].

The subjects of cabin noise in various flight vehicles and automobiles are more familiar in the investigation of fluid-structural coupled vibration of acoustoelastic enclosure, which mainly focus on characteristics of sound transmission through the elastic wall into interior sound field resulting from exterior air-borne sound [4]. In these cases, weak coupling has been commonly assumed because of the low density of air and high stiffness of cabin wall, which means that the cavity's interior sound pressure would have little influence on the vibration of cavity wall, and modals of interior sound field would also be affected very lightly [5]. In contrast, a much stronger coupling might be present when a water sound field takes place of the air [6].

The sound pressure is most commonly used to represent the property of sound field in the study of acoustic-structural coupling of acoustoelastic enclosure. The ratio of sound pressure at the outside surface of the elastic cavity wallboard to that at internal surface, which is defined as "noise reduction," is applied to evaluation of sound transmission characteristics $[4,7]$. Since the sound pressure would change greatly at different points of the sound field, the value of noise reduction would also be very different, and a comprehensive measure, for example, power flow, would be expected for an overall evaluation of vibration level of the enclosed sound field. The power flow has been validated and widely utilized as 
a comprehensive measure for evaluation of overall level of vibration energy of vibration isolation systems mounted on flexible foundations [8], which could also be explained as average sound power when applied to sound field analysis.

In this paper, through modal coupling analysis on the fluid-structural vibration of the water-filled enclosure with elastic boundaries, an efficient computational method is deduced to determine the vibratory power flow generated by exterior excitations on the outside surface of the elastic structure, including the total power flow entering into the fluid-structural coupled system and the net power flow transmitted into the hydroacoustic field. Characteristics of the coupled natural frequencies and modals are investigated by a numerical example of a rectangular water-filled cavity with five acoustic rigid walls and one elastic panel. Influential factors of power flow transmission characteristics are further discussed with the purpose of overall evaluation and reduction of the cavity water sound energy.

1.2. Theoretical Development. There has been a continuous effort for decades on investigation of fluid-structural mechanism of closed sound field with flexible boundaries. It has been recommended that the locally reactive acoustic normal impedance was the earlier theory to understand the sound absorption caused by the interaction between a reverberation room and its surrounding walls [9]. Later attention was paid to the modal coupling between the enclosed sound fields and the flexible walls to reveal the more complicated mechanism demonstrated by experimental results, which could not be interpreted by the locally reactive theory $[10,11]$.

The modal responses of acoustoelastic enclosures were first developed by Dowell et al. [12, 13] by applying Green's function to the inhomogeneous wave differential equation of the enclosed sound field and applying the classical modal and eigenvalue theorem to the simultaneous fluid-structural differential equations to result in a resolution of coupling modals. There are still other resolution methods for the same acoustoelasticity equations which could be referred to, such as Laplace transformation [14] and Ritz series [15]. In general, Dowell's method is based on the familiar uncoupled acoustic enclosure modes and structural modes, could be more easily implemented, and has been successfully applied to the investigation of variety of fluid-structural interaction systems $[16,17]$. Beginning with the "modal coupling method," Pan and Bies gave an insight analysis of the weak-coupled and well-coupled modals and their decay characteristics of a rectangular panel-cavity coupled system [18, 19]; Davis put forward a method for approximate estimation of the coupled natural frequencies of acoustoelastic enclosures by "coupling coefficient" [20].

Other important developments might lie in the field of discrete numerical techniques, such as FEM/BEM, for fluidstructural vibration analysis. However, these methods are usually preferred in the investigation of irregularly shaped cavities and targeting specific engineering problems. And that would be beyond the discussion of this paper, which would mainly focus on a general theoretical evaluation method for the overall vibration level of a fluid-filled enclosure through vibratory power flow calculation, especially based on Dowell's modal coupling theory.

\section{Theory}

2.1. Equations of Fluid-Structural Coupled Vibration. Consider that a fluid-filled enclosure occupies a volume $V$. Its boundary $D=D_{S}+D_{F}$, where $D_{F} \neq 0$ represents the flexible area of the surrounding wall and $D_{S}$ (might be zero) represents the acoustic rigid area.

The fluid inside the enclosure satisfied the wave equation and associated boundary condition.

$$
\begin{aligned}
K_{0} \nabla^{2} p(\sigma, t)-\rho_{0} \frac{\partial^{2} p(\sigma, t)}{\partial t^{2}} & =0 \quad(\sigma \in V) \\
\frac{\partial p(\sigma, t)}{\partial n} & =-\rho_{0} a_{n}(\sigma, t) \quad\left(\sigma \in D_{F}\right) \\
\frac{\partial p(\sigma, t)}{\partial n} & =0 \quad\left(\sigma \in D_{S}\right)
\end{aligned}
$$

where $p(\sigma, t)$ is the sound pressure at point $\sigma(x, y, z) \in V$; $a_{n}(\sigma, t)$ is the acceleration of the flexible wall in the normal direction $n$ (positive outward); $\rho_{0}$ and $K_{0}$ are the equilibrium fluid density and fluid volume stiffness, respectively.

If $D_{F}=0,(1)$ has modal solutions $F_{A r}(\sigma) \cdot \exp \left(j \omega_{A r} t\right), r=$ $0,1,2, \ldots$, where $\omega_{A r}$ is the $r$ th acoustical natural frequency in the condition of rigid boundary and $F_{A r}(\sigma)$ is the corresponding natural mode with orthogonality as follows:

$$
\begin{array}{r}
\iiint_{V} \frac{F_{A r}(\sigma) F_{A s}(\sigma)}{\rho_{0} c_{0}^{2}} \mathrm{~d} v= \begin{cases}0 & r \neq s \\
M_{A r} & r=s,\end{cases} \\
\iiint_{V} \frac{\left[\nabla F_{A r}(\sigma)\right]^{T} \cdot\left[\nabla F_{A s}(\sigma)\right]}{\rho_{0}} \mathrm{~d} v= \begin{cases}0 & r \neq s \\
\omega_{A r}^{2} M_{A r} & r=s,\end{cases}
\end{array}
$$

where $c_{0}=\sqrt{K_{0} / \rho_{0}}$ is the acoustic velocity of the fluid, $M_{A r}$ is the $r$ th acoustical modal mass in the condition of rigid boundary, and $\nabla F_{A r}(\sigma)=\left[\partial F_{A r} / \partial x, \partial F_{A r} / \partial y, \partial F_{A r} / \partial z\right]^{T}$ is the column gradient vector of modal function $F_{A r}(\sigma)$.

Consider the solution of (1) with $D_{F} \neq 0$ being in the form of modal superposition; that is,

$$
p(\sigma, t)=\sum_{r=0}^{\infty} F_{A r}(\sigma) P_{r}(t)=[\mathbf{F}(\sigma)]^{T} \mathbf{P}(t) \quad(\sigma \in V),
$$

where $\mathbf{F}(\sigma)$ and $\mathbf{P}(t)$ are column vectors of modal function $F_{r}(\sigma)$ and its corresponding modal coordinate $P_{r}(t)$, respectively; that is, $\mathbf{F}(\sigma)=\left[F_{A 0}(\sigma), F_{A 1}(\sigma), F_{A 2}(\sigma), \ldots\right]^{T}$ and $\mathbf{P}(t)=$ $\left[P_{0}(t), P_{1}(t), P_{2}(t), \ldots\right]^{T}$.

After substituting (5) into (1), multiply both sides of the resultant equation with a left-multiplication matrix (vector) $\mathbf{F}(\sigma)$ and finally integrating the equation over volume $V$, one obtains

$$
\begin{aligned}
\iiint_{V} \mathbf{F}(\sigma) & {\left[\nabla^{2} p(\sigma, t)\right] \mathrm{d} v } \\
- & \frac{1}{c_{0}^{2}} \iiint_{V}\left\{\mathbf{F}(\sigma)[\mathbf{F}(\sigma)]^{T} \ddot{\mathbf{P}}(t)\right\} \mathrm{d} v=0 .
\end{aligned}
$$


By applying Green's theorem to the first term of above equation, one has

$$
\begin{aligned}
\frac{1}{c_{0}^{2}} \iiint_{V}\left\{\mathbf{F}(\sigma)[\mathbf{F}(\sigma)]^{T} \ddot{\mathbf{P}}(t)\right\} \mathrm{d} v \\
\quad+\iiint_{V}[\nabla \mathbf{F}(\sigma)]^{T} \nabla \mathbf{F}(\sigma) \mathbf{P}(t) \mathrm{d} v \\
=\oiint_{D} \mathbf{F}(\sigma) \frac{\partial p(\sigma, t)}{\partial n} \mathrm{~d} A,
\end{aligned}
$$

where $\nabla \mathbf{F}(\sigma)$ is the gradient matrix of modal function $F_{r}(\sigma)$ : $\nabla \mathbf{F}(\sigma)=\left[\nabla F_{A 0}(\sigma), \nabla F_{A 1}(\sigma), \nabla F_{A 2}(\sigma), \ldots\right]$.

Now substitute the boundary condition equations (2) (3) and orthogonality equation (5) into (7):

$$
\mathbf{M}_{A}\left[\ddot{\mathbf{P}}(t)+\boldsymbol{\Omega}_{A}^{2} \mathbf{P}(t)\right]=-\oiint_{D} \mathbf{F}(\sigma) a_{n}(\sigma, t) \mathrm{d} A,
$$

where $\mathbf{M}_{A}$ and $\boldsymbol{\Omega}_{A}$ are diagonal matrices of acoustical modal masses and natural frequencies, respectively; that is, $\mathbf{M}_{A}=$ $\operatorname{diag}\left[M_{A 0}, M_{A 1}, M_{A 2}, \ldots\right]$ and $\boldsymbol{\Omega}_{A}=\operatorname{diag}\left[\omega_{A 0}, \omega_{A 1}, \omega_{A 2}, \ldots\right]$.

The flexible boundary of the cavity is assumed to be thinwall structures, where linear partial differential equations would be adopted to fit the thin-wall structures' vibration, such that

$$
\begin{array}{r}
S w(\sigma, t)+m_{B} \frac{\partial^{2} w(\sigma, t)}{\partial t^{2}}=p_{A}(\sigma, t)-p_{B}(\sigma, t) \\
\quad\left(\sigma \in D_{F}\right),
\end{array}
$$

where $S$ is a linear differential operator representing structural stiffness; $m_{B}$ is structural mass per unit area; $p_{A}(\sigma, t)$ and $p_{B}(\sigma, t)$ are excitations on the surface of the thin-wall structures due to the cavity acoustics and external dynamical forces (intensity of pressure), respectively; $w(\sigma, t)$ is the displacement response of the thin-wall structures, which is defined in the normal direction of $D_{F}$.

The solution of (9) could be expressed as

$$
w(\sigma, t)=\sum_{j=1}^{\infty} W_{B j}(\sigma) B_{j}(t)=[\mathbf{W}(\sigma)]^{T} \mathbf{B}(t)
$$

$$
\left(\sigma \in D_{F}\right),
$$

where $W_{B j}(\sigma)$ is the $j$ th modal function that is defined on $D_{F}$ and concerned with the property of the thin-wall structures in vacuo and $B_{j}(t)$ is the modal coordinate corresponding to $W_{B j}(\sigma) ; \mathbf{W}(\sigma)$ and $\mathbf{B}(t)$ are column vectors of $W_{B j}(\sigma)$ and $B_{j}(t)$, respectively; that is, $\mathbf{W}(\sigma)=\left[W_{B 1}(\sigma), W_{B 2}(\sigma)\right.$, $\left.W_{B 3}(\sigma), \ldots\right]^{T}$ and $\mathbf{B}(t)=\left[B_{1}(t), B_{2}(t), B_{3}(t), \ldots\right]^{T}$.

By substituting (10) into (9) and using the orthogonality of $W_{B j}(\sigma)$, there would be a modal differential function as follows:

$$
\mathbf{M}_{B}\left[\ddot{\mathbf{B}}(t)+\mathbf{\Omega}_{B}^{2} \mathbf{B}(t)\right]=\mathbf{Q}_{A}(t)+\mathbf{Q}_{B}(t),
$$

where $\mathbf{M}_{B}$ and $\boldsymbol{\Omega}_{B}$, expressed as $\mathbf{M}_{B}=\operatorname{diag}\left[M_{B 1}, M_{B 2}, M_{B 3}\right.$, $\ldots]$ and $\boldsymbol{\Omega}_{B}=\operatorname{diag}\left[\omega_{B 1}, \omega_{B 2}, \omega_{B 3}, \ldots\right]$, are diagonal matrices of the modal masses and natural frequencies respectively, and $\omega_{B j}$ and $M_{B j}=\iint_{D_{F}} m_{B}\left[W_{B j}(\sigma)\right]^{2} \mathrm{~d} A(j=1,2,3, \ldots)$ represent the $j$ th natural frequency and modal mass of the thin-wall structures in vacuo, respectively. $\mathbf{Q}_{A}(t)$ and $\mathbf{Q}_{B}(t)$ are column vectors of the general forces due to $p_{A}(\sigma, t)$ and $p_{B}(\sigma, t)$ loaded on the thin-wall structures in vacuo, respectively, and

$$
\begin{aligned}
& \mathbf{Q}_{A}(t)=\iint_{D_{F}} \mathbf{W}(\sigma) p_{A}(\sigma, t) \mathrm{d} A, \\
& \mathbf{Q}_{B}(t)=-\iint_{D_{F}} \mathbf{W}(\sigma) p_{B}(\sigma, t) \mathrm{d} A .
\end{aligned}
$$

The right-hand term of (8) and the first term $\mathbf{Q}_{A}(t)$ on the right hand of (11) are of the fluid-structural interaction between the sound field inside the cavity and its flexible walls. Substituting (10) into the right-hand term of (8) and taking notice of $a_{n}(\sigma, t)=\partial^{2} w(\sigma, t) / \partial t^{2}$ at $\sigma \in D_{F}$ and $a_{n}(\sigma, t)=0$ at $\sigma \in D_{S}$, one could define a coupling matrix $\mathbf{L}$ as follows:

$$
\begin{aligned}
& \mathbf{L}=\iint_{D_{F}} \mathbf{F}(\sigma)[\mathbf{W}(\sigma)]^{T} \mathrm{~d} A=\left[L_{r j}\right] \\
&(r=0,1,2, \ldots ; j=1,2,3, \ldots),
\end{aligned}
$$

where $L_{r j}$ denotes the element of the coupling matrix $\mathbf{L}$ at the $r$ th row and the $j$ th column.

And (8) turns into

$$
\mathbf{M}_{A}\left[\ddot{\mathbf{P}}(t)+\mathbf{\Omega}_{A}^{2} \mathbf{P}(t)\right]=-\mathbf{L} \cdot \ddot{\mathbf{B}}(t) .
$$

Dealing with $\mathbf{Q}_{A}(t)$, one could express $p_{A}(\sigma, t)$ in (12) with (5), and (11) would become

$$
\mathbf{M}_{B}\left[\ddot{\mathbf{B}}(t)+\boldsymbol{\Omega}_{B}^{2} \mathbf{B}(t)\right]=\mathbf{L}^{T} \cdot \mathbf{P}(t)+\mathbf{Q}_{B}(t) .
$$

2.2. Modal Analysis. In order to carry out a modal analysis about the fluid-structural vibration system governed by (14) and (15), let $\mathbf{Q}_{B}(t)=0$, and suppose that there exist vibration solutions as follows:

$$
\begin{aligned}
& \mathbf{P}(t)=\left(\sqrt{\mathbf{M}_{A}}\right)^{-1} \cdot \boldsymbol{\chi}_{A} \cdot \exp (j \omega t), \\
& \mathbf{B}(t)=\left(\boldsymbol{\Omega}_{B} \sqrt{\mathbf{M}_{B}}\right)^{-1} \cdot \boldsymbol{\chi}_{B} \cdot \exp (j \omega t),
\end{aligned}
$$

where $\sqrt{\mathbf{M}_{A}}=\operatorname{diag}\left\lfloor\sqrt{M_{A 0}}, \sqrt{M_{A 1}}, \sqrt{M_{A 2}}, \ldots\right\rfloor$ and $\sqrt{\mathbf{M}_{B}}=$ $\operatorname{diag}\left\lfloor\sqrt{M_{B 1}}, \sqrt{M_{B 2}}, \sqrt{M_{B 3}}, \ldots\right\rfloor$ are square roots of the diagonal acoustical modal matrix $\mathbf{M}_{A}$ and the diagonal structural modal matrix $\mathbf{M}_{B}$, respectively. $\chi_{A}=\left[\chi_{A 0}, \chi_{A 1}, \chi_{A 2}, \ldots\right]^{T}$ and $\chi_{B}=\left[\chi_{B 1}, \chi_{B 2}, \ldots\right]^{T}$ are column vectors of fluid-structural coupled modal shape coefficients related to the cavity sound field and the flexible boundary structures, respectively.

Substituting (16) into (14) and (15), an eigenvalue problem could be obtained as

$$
\mathbf{A} \boldsymbol{\chi}=\left[\begin{array}{ll}
\mathbf{A}_{11} & \mathbf{A}_{12} \\
\mathbf{A}_{21} & \mathbf{A}_{22}
\end{array}\right]\left[\begin{array}{l}
\chi_{A} \\
\chi_{B}
\end{array}\right]=\omega^{2}\left[\begin{array}{l}
\chi_{A} \\
\chi_{B}
\end{array}\right]=\omega^{2} \chi,
$$


where $\chi$ could be named as vector of fluid-structural coupled modal shape coefficients and $\mathbf{A}$ is a symmetric characteristic matrix, and A's partitioned matrices could be calculated by $\mathbf{A}_{11}=\mathbf{\Omega}_{A}^{2}+\left(\sqrt{\mathbf{M}_{A}}\right)^{-1} \mathbf{L M}_{B}^{-1} \mathbf{L}^{T}\left(\sqrt{\mathbf{M}_{A}}\right)^{-1}, \mathbf{A}_{12}=\mathbf{A}_{21}^{T}=$ $-\left(\sqrt{\mathbf{M}_{A}}\right)^{-1} \mathbf{L} \boldsymbol{\Omega}_{B}\left(\sqrt{\mathbf{M}_{B}}\right)^{-1}$, and $\mathbf{A}_{22}=\boldsymbol{\Omega}_{B}^{2}$.

Equation (17) would give eigenvalues of matrix $\mathbf{A}$, that is, $\omega_{C k}{ }^{2}=\Omega_{C k}{ }^{2}\left(1+j \eta_{C k}\right)$, and the accompanying eigenvectors $\left[\boldsymbol{\chi}^{(k)}\right]^{T}=\left[\left\{\boldsymbol{\chi}_{A}{ }^{(k)}\right\}^{T},\left\{\boldsymbol{\chi}_{B}{ }^{(k)}\right\}^{T}\right]=\left[\chi_{A 0}{ }^{(k)}, \chi_{A 1}{ }^{(k)}, \chi_{A 2}{ }^{(k)}, \ldots\right.$, $\left.\chi_{B 1}{ }^{(k)}, \chi_{B 2}{ }^{(k)}, \chi_{B 3}{ }^{(k)}, \ldots\right], k=0,1,2, \ldots$, where $\Omega_{C k}$ corresponds to the $k$ th fluid-structural natural frequency. $\eta_{C k}$ is the loss factor associated with the $k$ th damped normal mode, which might be resulting from the introduction of a complex stiffness of the flexible boundary or a complex volume stiffness $K_{0}$ of the fluid in consideration of the damping properties of the fluid-structural system. It should also be noticed that $\left[\chi^{(k)}\right]$ might be complex vectors when $\omega_{C k}{ }^{2}$ are complex numbers.

The fluid-structural coupled modal functions of the cavity's sound field and the flexible boundaries would be expressed as

$$
\begin{aligned}
& F_{C k}(\sigma)=[\mathbf{F}(\sigma)]^{T}\left(\sqrt{\mathbf{M}_{A}}\right)^{-1} \chi_{A}^{(k)} \quad(\sigma \in V), \\
& W_{C k}(\sigma)=[\mathbf{W}(\sigma)]^{T}\left(\boldsymbol{\Omega}_{B} \sqrt{\mathbf{M}_{B}}\right)^{-1} \chi_{B}^{(k)} \quad\left(\sigma \in D_{F}\right) .
\end{aligned}
$$

2.3. Vibratory Power Flow Transmission. In the condition that the flexible boundary structures of the cavity are subjected to a harmonic exterior excitation, that is, $p_{B}(\sigma, t)=P_{B}(\sigma)$. $\exp (j \omega t)$, let $\mathbf{P}_{B}=-\iint_{D_{F}} \mathbf{W}(\sigma) \cdot P_{B}(\sigma) \mathrm{d} A ; \mathbf{P}_{B}$ indicates that the amplitudes of general forces belong to the uncoupled flexible structures. The steady responses of the fluid-structural coupling cavity would be

$$
\begin{aligned}
p(\sigma, t) & =[\mathbf{F}(\sigma)]^{T}\left(\sqrt{\mathbf{M}_{A}}\right)^{-1} \mathbf{X}_{A} \mathbf{H T P}_{B} \exp (j \omega t) \\
& =P(\sigma, \omega) \cdot \exp (j \omega t) \quad(\sigma \in V) \\
w(\sigma, t) & =[\mathbf{W}(\sigma)]^{T}\left(\boldsymbol{\Omega}_{B} \sqrt{\mathbf{M}_{B}}\right)^{-1} \mathbf{X}_{B} \mathbf{H T P}_{B} \exp (j \omega t) \\
& =W(\sigma, \omega) \cdot \exp (j \omega t) \quad\left(\sigma \in D_{F}\right),
\end{aligned}
$$

where $P(\sigma, \omega)$ and $W(\sigma, \omega)$ denote the amplitudes of the harmonic sound pressure in the cavity and harmonic displacement of the thin-wall structures. $\mathbf{X}_{A}$ and $\mathbf{X}_{B}$ are matrices composed of arrays of eigenvectors of the characteristic matrix $\mathbf{A}$; that is, $\mathbf{X}_{A}=\left[\boldsymbol{\chi}_{A}{ }^{(0)}, \boldsymbol{\chi}_{A}{ }^{(1)}, \boldsymbol{\chi}_{A}{ }^{(2)}, \ldots\right]$ and $\mathbf{X}_{B}=$ $\left[\chi_{B}{ }^{(0)}, \chi_{B}{ }^{(1)}, \chi_{B}{ }^{(2)}, \ldots\right]$. And

$$
\begin{aligned}
\mathbf{H}(\omega) & =\left(-\omega^{2} \mathbf{M}_{C}+\mathbf{\Omega}_{C}{ }^{2} \cdot \mathbf{M}_{C}\right)^{-1} \\
\mathbf{T} & =-\mathbf{X}_{A}^{H}\left(\sqrt{\mathbf{M}_{A}}\right)^{-1} \mathbf{L M}_{B}^{-1}+\mathbf{X}_{B}^{H} \boldsymbol{\Omega}_{B}\left(\sqrt{\mathbf{M}_{B}}\right)^{-1},
\end{aligned}
$$

where $\mathbf{H}$ could be named as the complex frequency response matrix of the fluid-structural coupled cavity and $\mathbf{T}$ is a transformation matrix to transform the general force $\mathbf{P}_{B}$ into its fluid-structural expression (the derivation of the matrices $\mathbf{H}$ and $\mathbf{T}$ has been explained via (A.5) (A.8) in Appendix A.2). $\mathbf{M}_{C}=\mathbf{X}_{A}^{H} \cdot \mathbf{X}_{A}+\mathbf{X}_{B}^{H} \cdot \mathbf{X}_{B}$ is a diagonal matrix of the fluid-structural coupled modal masses, and $\boldsymbol{\Omega}_{C}=\operatorname{diag}\left[\omega_{C 0}, \omega_{C 1}, \omega_{C 2}, \ldots\right]$ is a diagonal matrix of the fluidstructural coupled natural frequencies. The superscript " $H$ " denotes Hermitian transposition of matrices.

The power flow (density) inputted by exterior excitation $p_{B}(\sigma, t)$ into the fluid-structural system is

$$
\begin{aligned}
p_{\text {in }}(\sigma, \omega)= & \frac{\omega}{2 \pi} \int_{0}^{\omega / 2 \pi} \operatorname{Re}\left\{p_{B}(\sigma, t)\right\} \\
& \cdot \operatorname{Re}\left\{-\frac{\partial w(\sigma, t)}{\partial t}\right\} \mathrm{d} t \\
= & -\frac{\omega}{2} \operatorname{Re}\left\{j W(\sigma, \omega) \cdot P_{B}^{*}(\sigma)\right\} \quad\left(\sigma \in D_{F}\right) .
\end{aligned}
$$

The total power flow input is

$$
\begin{gathered}
P_{\text {in }}(\omega)=\iint_{D_{F}} p_{\text {in }}(\sigma, t) \mathrm{d} A=-\frac{\omega}{2} \operatorname{Re}\left\{j \mathbf{P}_{B}^{T} \mathbf{T}^{T} \mathbf{H}(\omega)\right. \\
\left.\cdot \mathbf{X}_{B}^{T}\left(\boldsymbol{\Omega}_{B} \sqrt{\mathbf{M}_{B}}\right)^{-1} \iint_{D_{F}} \mathbf{W}(\sigma) \cdot P_{B}^{*}(\sigma) \mathrm{d} A\right\}=\frac{\omega}{2} \\
\cdot \operatorname{Re}\left\{j \mathbf{P}_{B}^{T} \mathbf{T}^{T} \mathbf{H}(\omega) \cdot \mathbf{X}_{B}^{T}\left(\boldsymbol{\Omega}_{B} \sqrt{\mathbf{M}_{B}}\right)^{-1} \mathbf{P}_{B}^{*}\right\},
\end{gathered}
$$

where the superscript “*” denotes conjugation of complex numbers.

The power flow (density) transmitted through the fluidstructural interaction boundary of the cavity into the enclosed sound field is

$$
\begin{aligned}
p_{\text {tr }}(\sigma, \omega)= & \frac{\omega}{2 \pi} \int_{0}^{\omega / 2 \pi} \operatorname{Re}\left\{\left.p(\sigma, t)\right|_{\sigma \in D_{F}}\right\} \\
& \cdot \operatorname{Re}\left\{-\left.\frac{\partial w(\sigma, t)}{\partial t}\right|_{\sigma \in D_{F}}\right\} \mathrm{d} t \\
= & \frac{\omega}{2} \operatorname{Re}\left\{\left.j W^{*}(\sigma, \omega) \cdot P(\sigma, \omega)\right|_{\sigma \in D_{F}}\right\} .
\end{aligned}
$$

The total transmission power flow is

$$
\begin{aligned}
P_{\mathrm{tr}}(\omega) & =\iint_{D_{F}} p_{\operatorname{tr}}(\sigma, \omega) \mathrm{d} A \\
& =\frac{\omega}{2} \operatorname{Re}\left\{\iint_{D_{F}}[\mathbf{F}(\sigma)]^{T} \cdot \boldsymbol{\Lambda}(\omega) \cdot \mathbf{W}(\sigma) \mathrm{d} A\right\} \\
& =\frac{\omega}{2} \operatorname{Re}\left\{\sum_{r=0}^{\infty} \sum_{j=1}^{\infty} L_{r j} \cdot \lambda_{r j}(\omega)\right\},
\end{aligned}
$$

where $\quad \boldsymbol{\Lambda}(\omega) \quad=\quad j\left(\sqrt{\mathbf{M}_{A}}\right)^{-1} \mathbf{X}_{A} \mathbf{H}(\omega)$. $\mathbf{T P}_{B} \mathbf{P}_{B}^{H} \mathbf{T}^{H}[\mathbf{H}(\omega)]^{*} \mathbf{X}_{B}^{H}\left(\boldsymbol{\Omega}_{B}^{*} \sqrt{\mathbf{M}_{B}}\right)^{-1}=\left[\lambda_{r j}(\omega)\right]$ might be named as a power transmission matrix and $\lambda_{r j}(\omega)$ denotes the element of matrix $\Lambda(\omega)$ at the $r$ th row and the $j$ th column. 


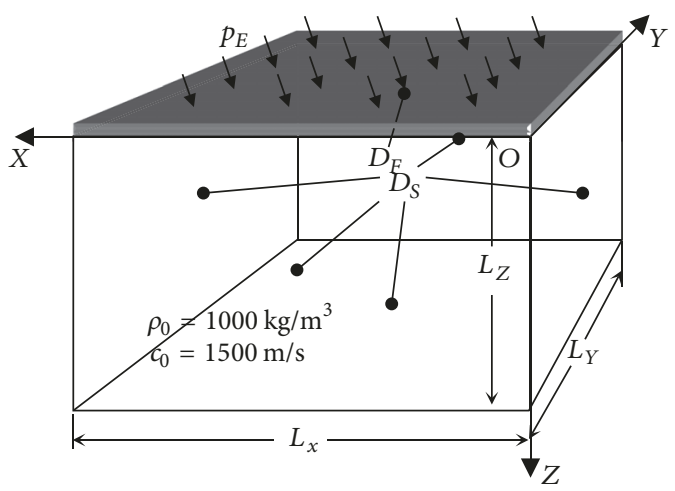

FIGURE 1: A panel-cavity coupled system.

\section{Numerical Simulation and Analysis}

3.1. Simulation Model. A panel-cavity coupled system shown in Figure 1 consists of a rectangular water-filled room with five rigid walls and one simply supported plate subject to exterior harmonic distributed force (pressure) $p_{E}(\sigma, t)$.

In discussion of the distribution shape of exterior excitation, the plane harmonic wave incident is a common assumption. Suppose that $p_{E}(\sigma, t)=P_{E} \cdot \exp \left[j\left(\omega t-k^{\prime} x \sin \theta\right)\right]$, where $P_{E}$ is the amplitude, $k^{\prime}$ is the wave number, and $\theta$ is the incident angle ( $p_{E}$ is uniform along the $y$ direction); $p_{E}$ has a perpendicular component $p_{B}(\sigma, t)=P_{E} \cos \theta$. $\exp \left(-j k^{\prime} x \sin \theta\right) \cdot \exp (j \omega t)=P_{B}(\sigma) \cdot \exp (j \omega t)$. Generally, $P_{B}(\sigma)$ would be a complex function and have infinite variety of distribution shapes when the wave frequency, velocity, and incident angle changed. Figure 2 shows one example of distribution shape of $P_{B}(\sigma)$ with wave frequency $f=500 \mathrm{~Hz}$, velocity $c=344 \mathrm{~m} / \mathrm{s}$, and incident angle $\theta=\pi / 3$. It is true that the modal coupling method is valid in dealing with those variant distribution shapes of $P_{B}(\sigma)$. However, some specific analysis on the special case with $\theta=0$, that is, a uniform $P_{B}$ over the plate surface, would also give indications of general significance. The uniform excitation had been adopted by other authors previously $[7,15]$. And, moreover, taking sonar array cavities as examples, they are regularly mounted on related ship structures through rubber blankets; the uniform structural excitation assumption would be a basic consideration.

For the water-filled rectangular room, the natural frequencies and modal functions of the sound field with rigid boundaries are determined by

$$
\begin{aligned}
& \omega_{A r}^{2}=\frac{K_{0}}{\rho_{0}}\left[\left(\frac{m_{A r} \pi}{L_{x}}\right)^{2}+\left(\frac{n_{A r} \pi}{L_{y}}\right)^{2}+\left(\frac{l_{A r} \pi}{L_{z}}\right)^{2}\right] \\
& F_{A r}(x, y, z) \\
& \quad=\cos \left(\frac{m_{A r} \pi x}{L_{x}}\right) \cos \left(\frac{n_{A r} \pi y}{L_{y}}\right) \cos \left(\frac{l_{A r} \pi z}{L_{z}}\right),
\end{aligned}
$$

where $\forall\left(m_{A r}, n_{A r}, l_{A r}\right) \in N^{3}, r \in N$, and let $\omega_{A r}$ arrange in a sequence $\left|\omega_{A 0}{ }^{2}\right|<\left|\omega_{A 1}{ }^{2}\right|<\left|\omega_{A 2}{ }^{2}\right|<\cdots$.
For a simply supported plate, its natural frequencies and modal functions are determined by

$$
\begin{aligned}
\omega_{B j}^{2} & =\frac{\pi^{4} E h^{2}}{12 \rho\left(1-\mu^{2}\right)}\left(\frac{m_{B j}^{2}}{L_{x}^{2}}+\frac{n_{B j}^{2}}{L_{y}^{2}}\right)^{2} \\
W_{B j}(x, y, 0) & =\sin \left(\frac{m_{B j} \pi x}{L_{x}}\right) \sin \left(\frac{n_{B j} \pi y}{L_{y}}\right),
\end{aligned}
$$

where $E, \rho, h$, and $\mu$ are Young's modulus, mass density, thickness, and Poisson's ratio of the plate, respectively; $\forall\left(m_{B j}, n_{B j}\right) \in N^{2}, j \in N_{+}$, let $\omega_{B j}$ arrange in a sequence $\left|\omega_{B 1}{ }^{2}\right|<\left|\omega_{B 2}{ }^{2}\right|<\left|\omega_{B 3}{ }^{2}\right|<\cdots$.

The geometrical and material properties adopted for numerical computation are as follows: $L_{x}=0.4 \mathrm{~m}, L_{y}=$ $0.6 \mathrm{~m}, L_{z}=0.7 \mathrm{~m}$, and $h=0.005 \mathrm{~m} ; \rho_{0}=1000 \mathrm{~kg} / \mathrm{m}^{3}$ and $K_{0}=2.25 \times 10^{9} \mathrm{~Pa} \times\left(1+10^{-4} \mathrm{j}\right) ; E=2.0 \times 10^{11} \mathrm{~Pa} \times\left(1+10^{-3} \mathrm{j}\right)$, $\rho=7.8 \times 10^{3} \mathrm{~kg} / \mathrm{m}^{3}$, and $\mu=0.28$ (steel).

3.2. Modal Analysis. There is a convergence investigation about the fluid-structural coupled natural frequencies resulting from (17) at first. In Table 1, with a fixed number of plate modals involved in calculation, the convergence of coupled natural frequencies could be observed by increasing the number of water sound modals involved. The convergence could also be observed by increasing the number of plate modals involved in Table 2 . It could be suggested that the modal coupling method could achieve good convergence in solving the fluid-structural coupled problem described here. And it could also be observed that the convergence at lower frequencies is more rapid than that at higher frequencies, and the solution precision would be more dependent on accounting for more water sound modals. However, there is no need to carry out a high accuracy calculation here for a theoretical qualitative analysis, and in the later part of this paper, 50 plate modals and 500 water sound field modals are taken into consideration, by which totally 550 coupled modals could be revealed. And also, because there is no need to list all 550 modes here, only partial data (the first several modes) are listed in Tables 1 and 2

Figure 3 gives a comparison of sound pressure solutions between the modal coupling approach and FEM (by the software of LMS Virtual.Lab Acoustics) as a theoretical validation verification. The differences between the two results in Figure 3(a) are due to modal truncation; more modes are involved in the FEM/BEM software.

The fluid-structural coupled natural frequencies resulting from (17) are compared with those of sound field with rigid boundaries obtained by (27) and simply supported steel plate obtained by (29), which are listed in Table 3. As mentioned above, 550 coupled modals have been obtained by involving 50 plate modals and 500 water sound field modals in modal coupling calculation, but only the first 11 coupled modal frequencies are presented.

As a whole, it could be concluded that the fluid-structural frequencies are very different from those of the water sound field (with rigid boundaries) and the flexible boundary plate (in vacuo), which means that there would be a strong 
TABLE 1: Coupled natural frequencies with different number of water sound modals involved.

\begin{tabular}{|c|c|c|c|c|c|}
\hline \multirow[b]{2}{*}{ Number of water sound modals involved } & \multicolumn{5}{|c|}{ Fluid-structural coupled natural frequency $f_{C k}(\mathrm{~Hz})$} \\
\hline & 50 & 200 & 500 & 1000 & 2000 \\
\hline Number of plate modals involved & 50 & 50 & 50 & 50 & 50 \\
\hline \multicolumn{6}{|l|}{$k$} \\
\hline 0 & 0 & 0 & 0 & 0 & 0 \\
\hline 1 & 106.8 & 103.1 & 101.5 & 100.9 & 100.3 \\
\hline 2 & 218.5 & 208.4 & 206.0 & 203.9 & 202.5 \\
\hline 3 & 234.4 & 222.7 & 217.6 & 215.5 & 213.4 \\
\hline 4 & 291.1 & 270.9 & 264.7 & 262.2 & 259.7 \\
\hline 5 & 447.6 & 415.1 & 404.7 & 400.2 & 395.9 \\
\hline 6 & 455.4 & 420.7 & 409.1 & 404.5 & 399.8 \\
\hline 7 & 476.1 & 457.3 & 451.8 & 447.1 & 443.9 \\
\hline 8 & 561.9 & 543.1 & 537.2 & 533.4 & 530.5 \\
\hline 9 & 626.7 & 581.5 & 566.2 & 559.0 & 552.3 \\
\hline 10 & 682.5 & 617.7 & 599.4 & 592.1 & 584.7 \\
\hline
\end{tabular}

TABLE 2: Coupled natural frequencies with different number of plate modals involved.

\begin{tabular}{|c|c|c|c|c|c|}
\hline \multirow[b]{2}{*}{ Number of water sound modals involved } & \multicolumn{5}{|c|}{ Fluid-structural coupled natural frequencies $f_{C k}(\mathrm{~Hz})$} \\
\hline & 500 & 500 & 500 & 500 & 500 \\
\hline Number of plate modals involved & 25 & 50 & 100 & 200 & 500 \\
\hline \multicolumn{6}{|l|}{$k$} \\
\hline 0 & 0 & 0 & 0 & 0 & 0 \\
\hline 1 & 101.5 & 103.1 & 101.5 & 101.5 & 101.5 \\
\hline 2 & 206.1 & 208.4 & 206.0 & 206.0 & 206.0 \\
\hline 3 & 217.6 & 222.7 & 217.5 & 217.5 & 217.5 \\
\hline 4 & 264.7 & 270.9 & 264.7 & 264.7 & 264.7 \\
\hline 5 & 404.7 & 415.1 & 404.6 & 404.6 & 404.6 \\
\hline 6 & 409.2 & 420.7 & 409.1 & 409.1 & 409.1 \\
\hline 7 & 451.8 & 457.3 & 451.6 & 451.6 & 451.6 \\
\hline 8 & 537.2 & 543.1 & 537.1 & 537.1 & 537.1 \\
\hline 9 & 566.2 & 581.5 & 566.0 & 566.0 & 566.0 \\
\hline 10 & 599.5 & 617.7 & 599.3 & 599.3 & 599.3 \\
\hline
\end{tabular}

TABLE 3: Natural frequencies of the water sound field, plate, and fluid-structural coupled cavity.

\begin{tabular}{|c|c|c|c|c|c|c|c|}
\hline \multicolumn{3}{|c|}{ Natural frequencies of water sound field } & \multicolumn{3}{|c|}{ Natural frequencies of plate } & \multicolumn{2}{|c|}{$\begin{array}{c}\text { Fluid-structural coupled } \\
\text { natural frequencies }\end{array}$} \\
\hline$r$ & $\left(m_{A r}, n_{A r}, l_{A r}\right)$ & $f_{A r}(\mathrm{~Hz})$ & $j$ & $\left(m_{B j}, n_{B j}\right)$ & $f_{B j}(\mathrm{~Hz})$ & $k$ & $f_{C k}(\mathrm{~Hz})$ \\
\hline 0 & $(0,0,0)$ & 0 & & & & 0 & 0 \\
\hline 1 & $(0,0,1)$ & 1071.4 & 1 & $(1,1)$ & 108.0 & 1 & 101.5 \\
\hline 2 & $(0,1,0)$ & 1250.0 & 2 & $(1,2)$ & 207.6 & 2 & 206.0 \\
\hline 3 & $(0,1,1)$ & 1646.3 & 3 & $(2,1)$ & 332.2 & 3 & 217.6 \\
\hline 4 & $(1,0,0)$ & 1875.0 & 4 & $(1,3)$ & 373.7 & 4 & 264.7 \\
\hline 5 & $(0,0,2)$ & 2142.9 & 5 & $(2,2)$ & 431.9 & 5 & 404.7 \\
\hline 6 & $(1,0,1)$ & 2159.5 & 6 & $(2,3)$ & 598.0 & 6 & 409.1 \\
\hline 7 & $(1,1,0)$ & 2253.5 & 7 & $(1,4)$ & 606.3 & 7 & 451.8 \\
\hline 8 & $(0,1,2)$ & 2480.8 & 8 & $(3,1)$ & 705.9 & 8 & 537.2 \\
\hline 9 & $(1,1,1)$ & 2495.2 & 9 & $(3,2)$ & 805.6 & 9 & 566.2 \\
\hline 10 & $(0,2,0)$ & 2500.0 & 10 & $(2,4)$ & 830.5 & 10 & 599.4 \\
\hline
\end{tabular}




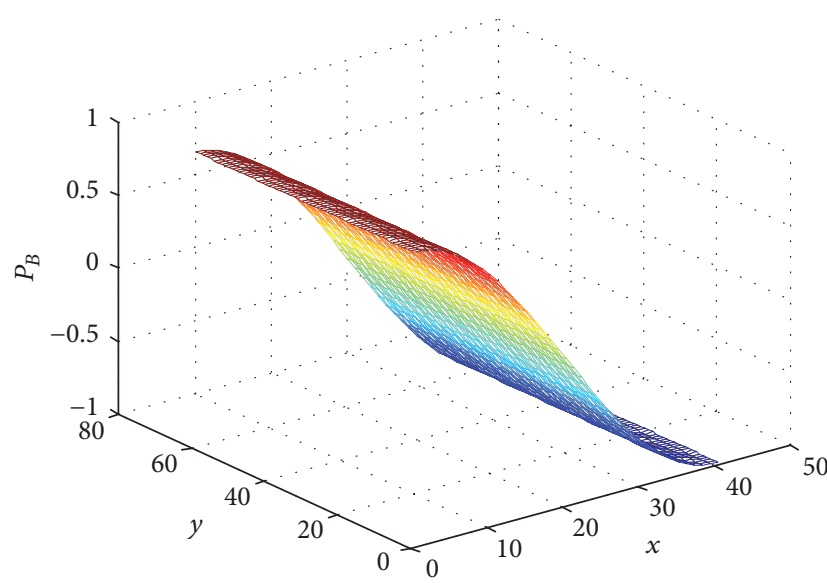

(a) Real part

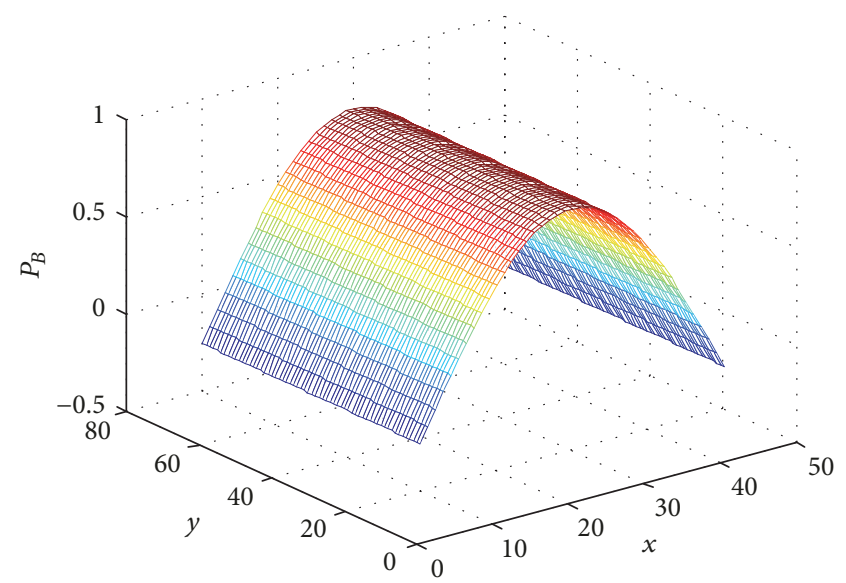

(b) Imaginary part

FIGURE 2: One example of nonuniform distribution shape of $P_{B}(\sigma)$ (wave frequency $f=500 \mathrm{~Hz}$, velocity $c=344 \mathrm{~m} / \mathrm{s}$, and incident angle $\theta=\pi / 3)$.

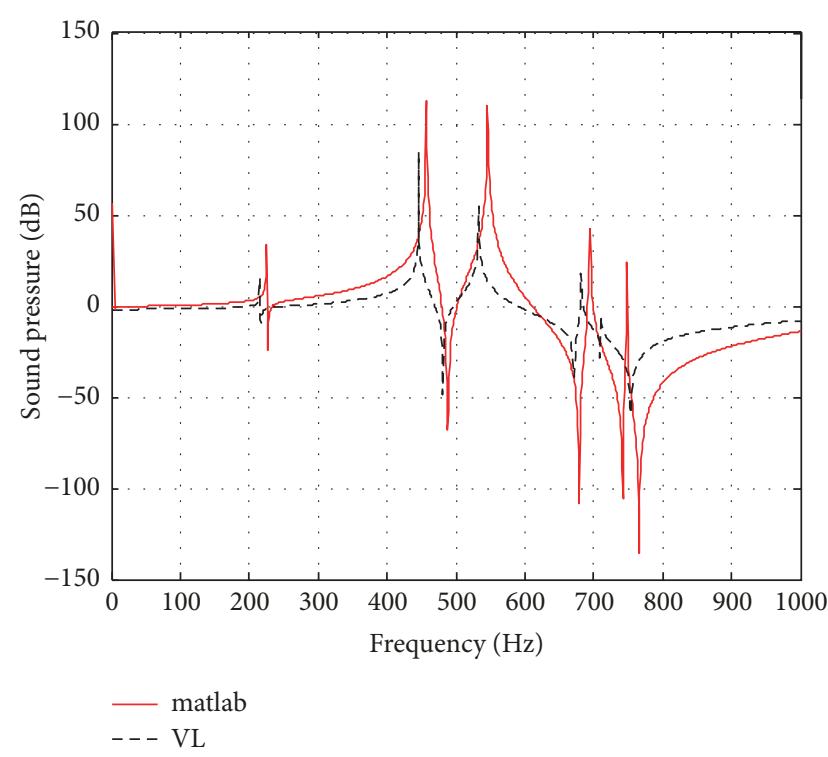

(a) Comparison of sound pressure solutions

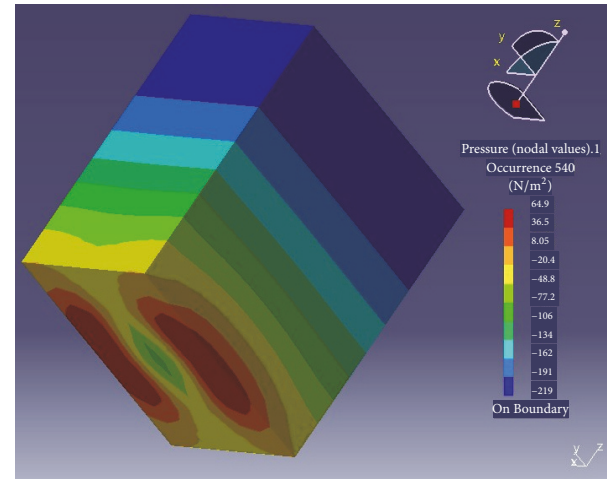

(b) Cloud picture of boundary sound pressure by VL $(540 \mathrm{~Hz})$

FIGURE 3: Comparison of sound pressure solutions between the modal coupling approach and FEM.

coupling between the enclosed water sound field and its elastic surrounding structures. If the fluid in the cavity was $\operatorname{air}\left(\rho_{0} \approx 1.29 \mathrm{~kg} / \mathrm{m}^{3} ; c_{0} \approx 344 \mathrm{~m} / \mathrm{s}\right)$, it could be found that the coupled natural frequencies $f_{C k}$ would approximately be equal to either some uncoupled structural natural frequencies $f_{B j}$ or some uncoupled cavity acoustical natural frequencies $f_{A r}$, and that is a situation of weak coupling. The motion of each subsystem in a weakly coupled system will not be essentially different from that of the uncoupled systems. However, if the density of the medium in the cavity is much denser than air, such as water, the coupling may turn out to be strong, and big deformation of the resulting modes from the uncoupled panel and the cavity modes may be expected [18]. In this sense, the strong coupling could be judged by any unneglectable departure of coupled natural frequencies from every natural frequency of the uncoupled flexible structures and cavity.

Except $f_{A 0}=0$, the natural frequencies of the water sound field are much higher than those of the simply supported plate, and the fluid-structural coupled natural frequencies are inclined to come to be rather lower. It seemed that one might carry out a comparison between the coupled natural frequencies and those of plate, and the change regulation of differences of adjacent fluid-structural natural frequencies $f_{C(k+1)}-f_{C k}$ is similar to those $f_{B j}-f_{B(j-1)}$ of the plate. However, the frequency distribution of coupled natural frequencies would turn to be lower and more crowded as the order $k$ increased. 


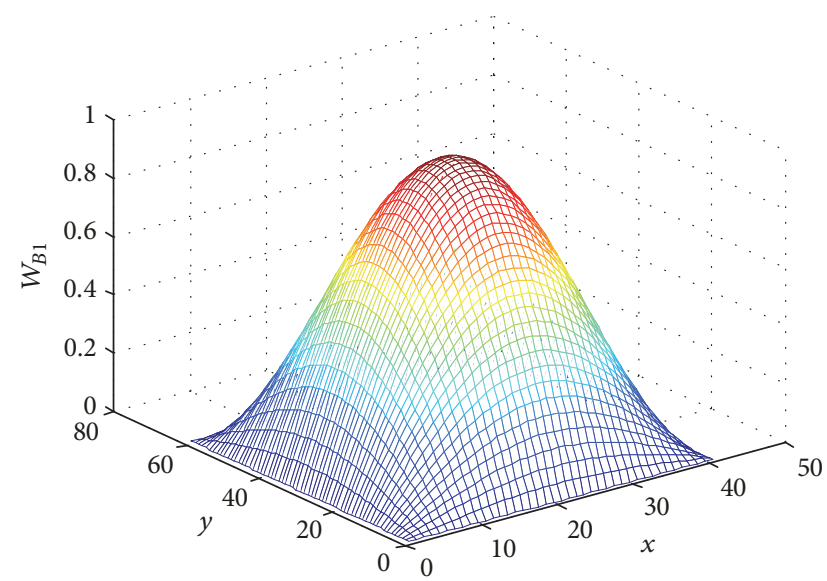

(a) $W_{B 1}=\sin \left(\pi x / L_{x}\right) \sin \left(\pi y / L_{y}\right), f_{B 1} \approx 108.0 \mathrm{~Hz}$

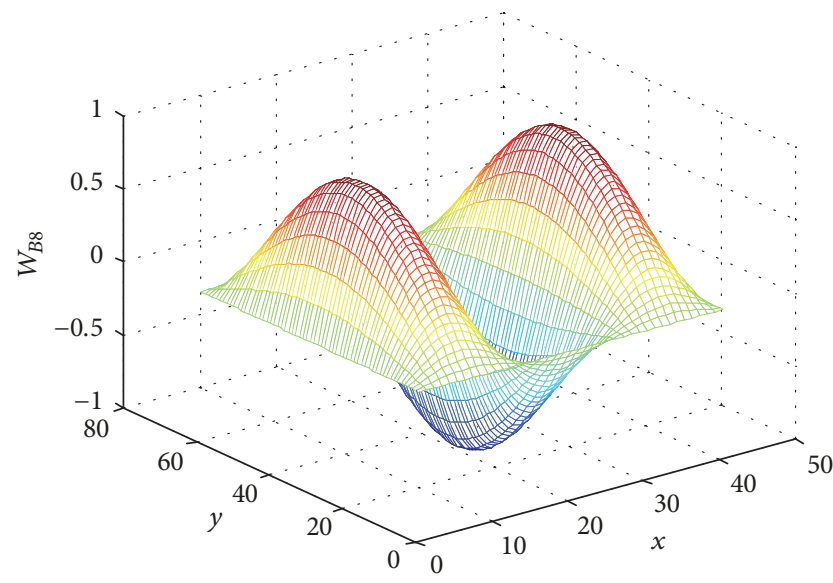

(c) $W_{B 8}=\sin \left(3 \pi x / L_{x}\right) \sin \left(\pi y / L_{y}\right), f_{B 4} \approx 705.9 \mathrm{~Hz}$

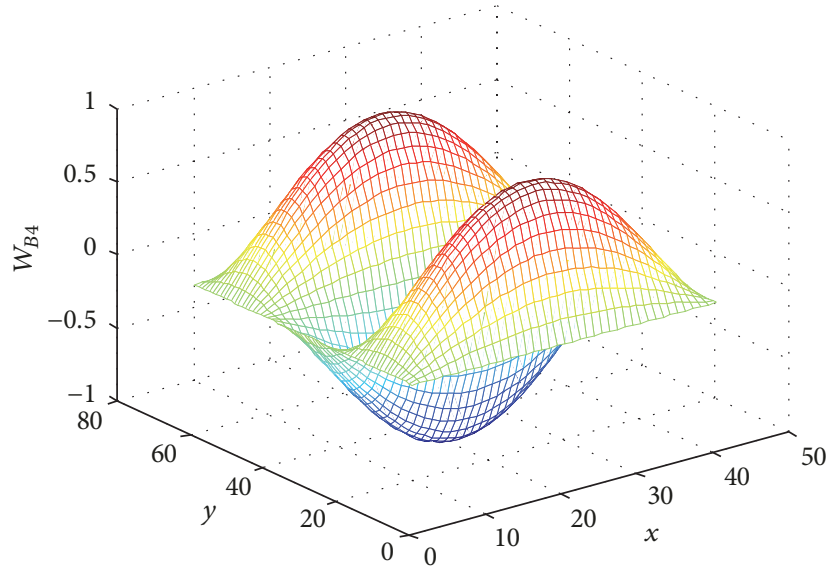

(b) $W_{B 4}=\sin \left(\pi x / L_{x}\right) \sin \left(3 \pi y / L_{y}\right), f_{B 4} \approx 373.4 \mathrm{~Hz}$

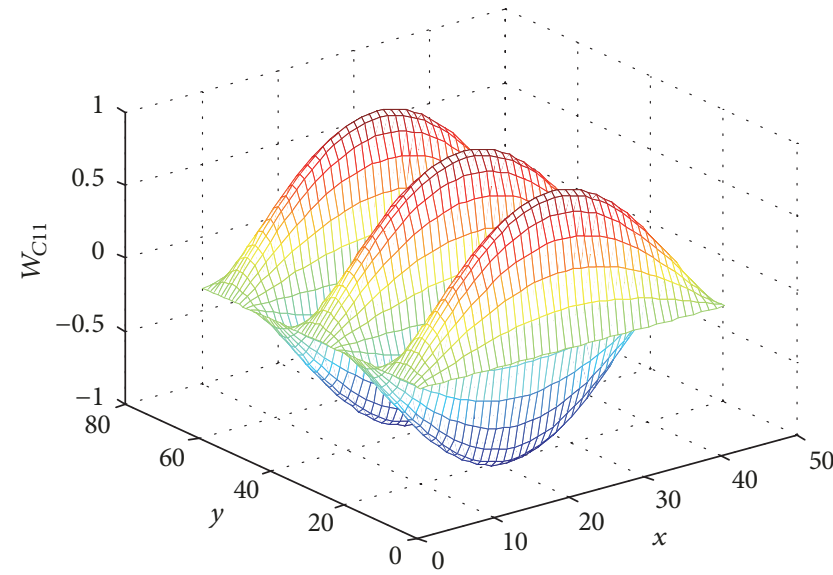

(d) $W_{B 11}=\sin \left(\pi x / L_{x}\right) \sin \left(5 \pi y / L_{y}\right), f_{B 11} \approx 905.2 \mathrm{~Hz}$

FIGURE 4: Some uncoupled plate modals.

In order to reveal modal coupling characteristics, Figures 4 and 5 show several uncoupled plate modals $W_{B j}(\sigma)$ which are expressed by (30) and fluid-structural coupled plate modals $W_{C k}(\sigma)$ which are determined by (29) through modal coupling calculation. The figure shows that $W_{C 0}$ is almost the same as $W_{B 1}$, while it is true according to $W_{C 0}$ 's expression and that is just an example of weak-coupled modal. It seemed that the coupled modal shape $W_{C 3}$ is similar to the uncoupled modal shape $W_{B 4}$; however, they are quite different in fact according to the expression of $W_{C_{3}}$, and that is a strong coupled modal. Phenomena of strong modal coupling are obvious when inspecting $W_{C 7}$ and $W_{C 8}$ shown in Figure 5.

The similar couplings have also happened to the acoustical cavity modals. And, moreover, except that the coupled acoustical modal $F_{C 0}$ is practically equal to $F_{A 0}$, that is, the rigid body modal of the uncoupled cavity sound field, all other fluid-structural coupled acoustical cavity modals are composed in a strong coupling manner; that is, they are linear combinations of several $F_{A r}$, the uncoupled acoustical modals of the rigid wall cavity. As $F_{C r}(\sigma)$ is defined in three-dimensional space and it is inconvenient to plot it by a planar figure, Figure 6(a) illustrates one coupled acoustical modal shape in the plane $z=L_{z} / 2$, and Figure 6(b) illustrates the appurtenant participant coefficients of the uncoupled acoustical cavity modals in the constitution of coupled acoustical cavity modal.

3.3. Power Flow Transmission. In the simulation model of Figure 1, the vibratory power flow inputted by exterior excitation into the whole fluid-structural coupled system and the enclosed water sound field, that is, $P_{\text {in }}$ calculated by (24) and $P_{\mathrm{tr}}$ calculated by (26), is dissipated by system damping. And thus the higher or lower power flow level would be a comprehensive indicator to measure the vibration level or energy level of the panel-cavity coupled system and water sound field.

Figure 7 shows the spectrum of input power flow $P_{\text {in }}$ and transmitted power flow $P_{\mathrm{tr}}$, in which the drop between $P_{\text {in }}$ and $P_{\mathrm{tr}}$ is the dissipation power of the plate's damping. Because the exterior excitation is symmetric (uniform $P_{B}$ as mentioned in Section 3.1), only symmetric modals are present, and the spectrum peaks at $0 \mathrm{~Hz}, 217.6 \mathrm{~Hz}, 451.8 \mathrm{~Hz}$, and $537.2 \mathrm{~Hz}$ could be associated with the modals shown in Figures 5 and 6. There is some similarity between $P_{\text {in }}$ or $P_{\operatorname{tr}}$ and the water 


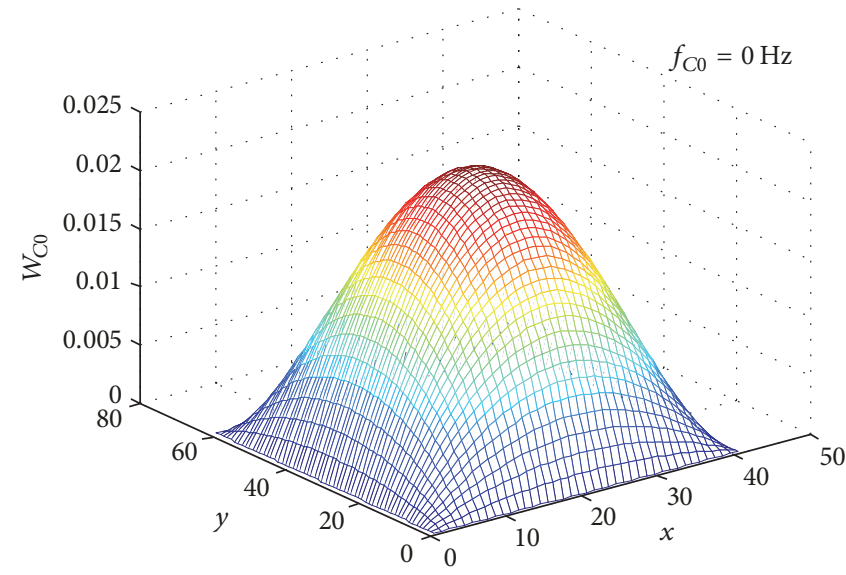

(a) $W_{C 0} \approx 0.0248\left(W_{B 1}+0.05 W_{B 4}+0.02 W_{B 8}+0.01 W_{B 11}\right)$

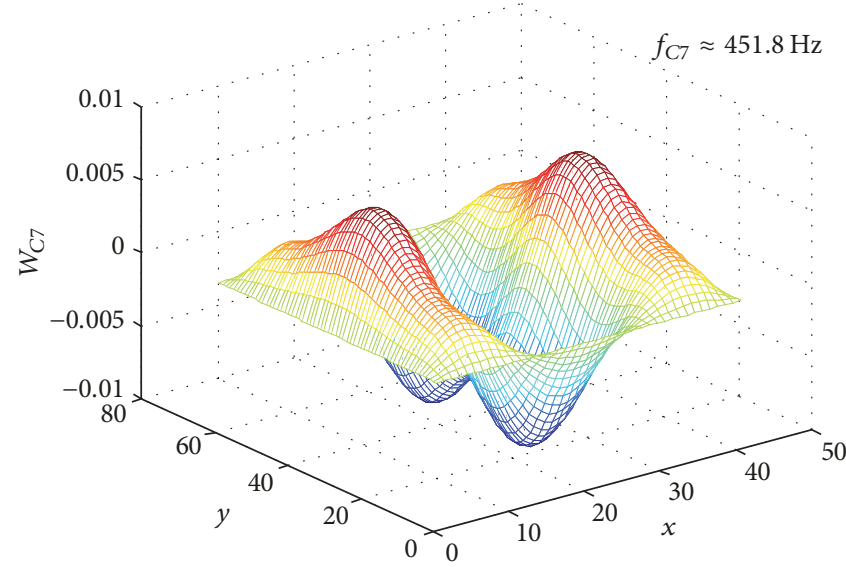

(c) $W_{C 7} \approx 0.0074\left(-0.28 W_{B 1}-0.26 W_{B 4}+W_{B 8}+0.14 W_{B 11}+0.07 W_{B 12}\right)$

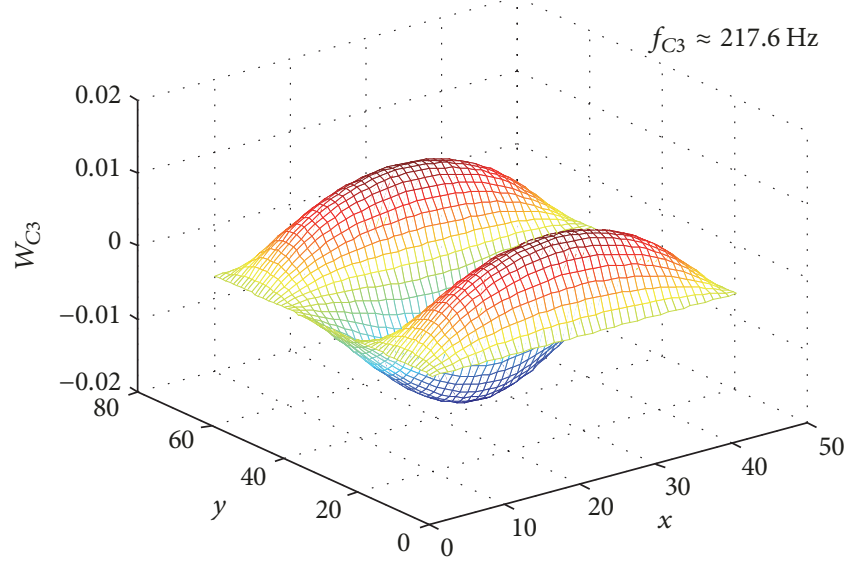

(b) $W_{C 3} \approx 0.0132\left(-0.21 W_{B 1}+W_{B 4}+0.04 W_{B 8}+0.05 W_{B 11}+0.03 W_{B 12}\right)$

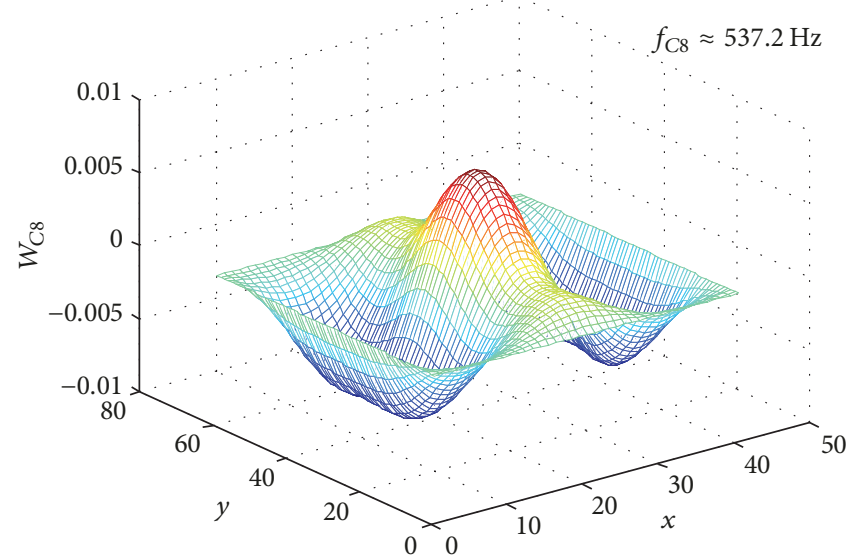

(d) $W_{C 8} \approx 0.0062\left(-0.11 W_{B 1}-0.16 W_{B 4}-W_{B 8}+0.18 W_{B 11}+0.08 W_{B 12}\right)$

FIGURE 5: Some coupled plate modals.

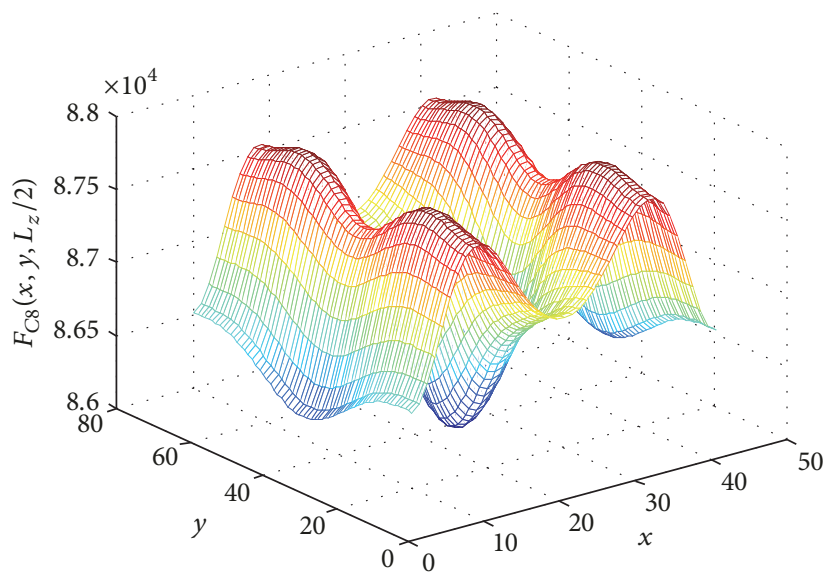

(a) $F_{C 8}\left(x, y, L_{z / 2}\right), f_{C 8} \approx 537.2 \mathrm{~Hz}$

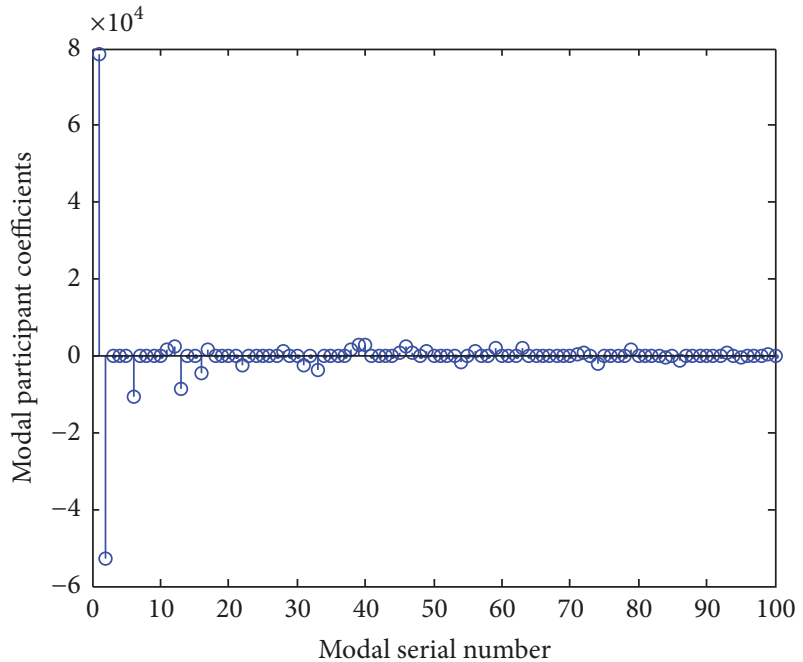

(b) Participant coefficients of the uncoupled cavity modals

FIGURE 6: Simple illustration of one coupled cavity modal shape. 


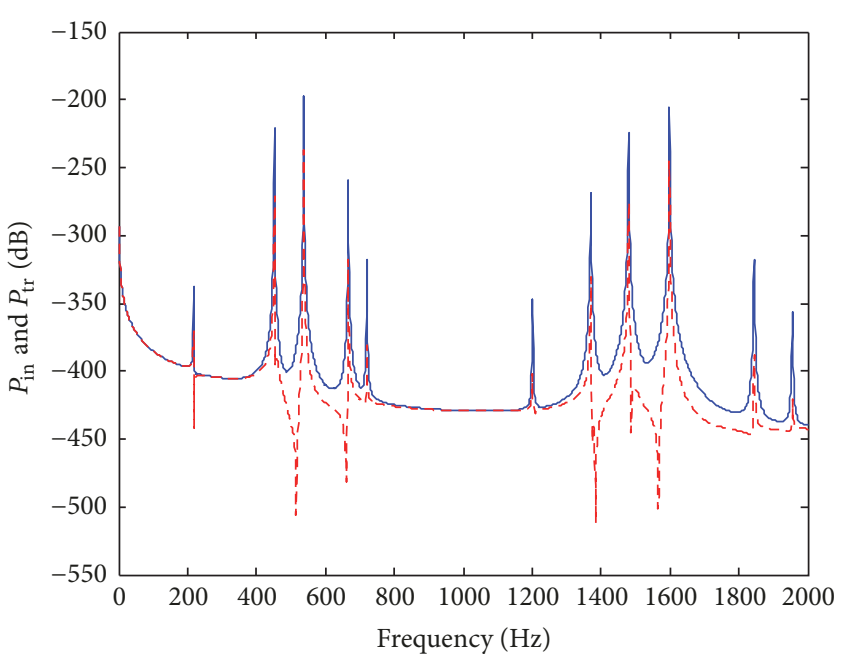

$-P_{\text {in }}$
$---P_{\text {tr }}$

FIgURE 7: Spectrum of $P_{\text {in }}$ and $P_{\text {tr }}$.

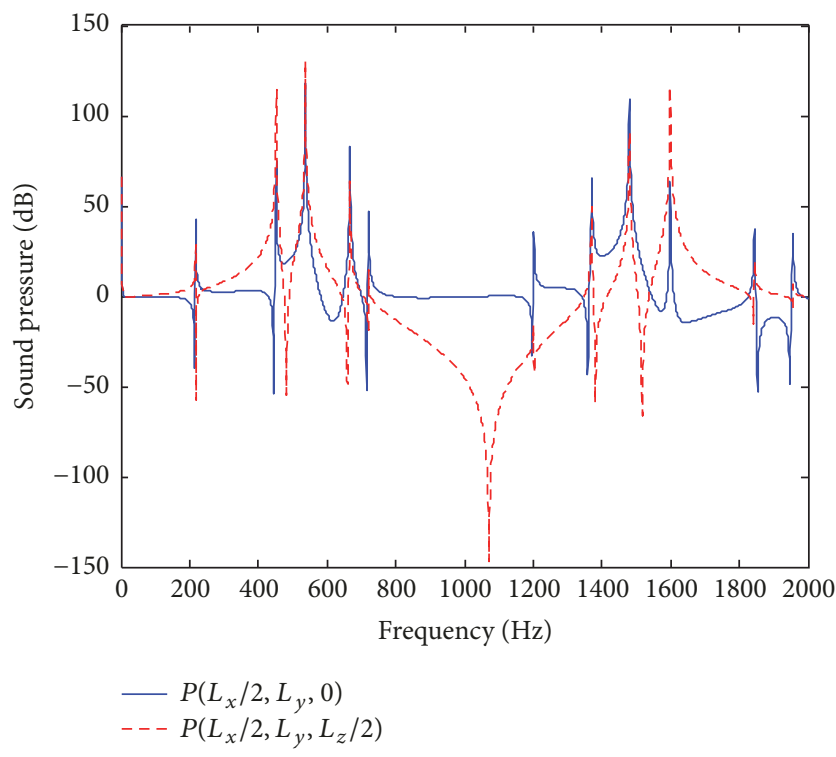

Figure 8: Spectrum of water sound pressure.

sound pressure at the center of the plate's interior surface, that is, $P\left(\left(L_{x} / 2, L_{y} / 2,0\right), \omega\right)$ (refer to $\left.(21)\right)$, which is shown in Figure 8 . However, the power flow is of evaluation of sound power.

As a theoretical investigation, hypothesize that the material property parameters of the plate, that is, Young's modulus $E$, mass density $\rho$, Poisson's ratio $\mu$, and damping loss factor, could be altered independently. In Figure 9(a), transmitted power flows are compared under different Young's modulus of the elastic plate, where the value of $7.24 \times 10^{10} \mathrm{~Pa}$ is by reference to aluminium. When the plate's elasticity modulus decreases, the plate's natural frequencies would decrease simultaneously, and this would move the fluid-structural coupled natural frequencies into lower frequency ranges, which has been predicted by Table 3. Thus the alteration of elasticity modulus of plate would lead to a phenomenon of "frequency shifting." The $P_{\mathrm{tr}}$ spectrum with smaller plate's elasticity modulus could be regarded as a contraction of that with greater elasticity modulus toward lower frequencies, and the peaks of $P_{\operatorname{tr}}$ would occur at relatively lower frequencies and become more crowded. However, since the variation range of Young's modulus would be limited in practice, its influence on power flow transmission might not be very serious, and it could also be observed that reduction of Young's modulus might bring about a benefit of slight reduction of $P_{\mathrm{tr}}$ 's peak valleys.

In order to make an inspection of the alteration of differences between $P_{\text {in }}$ and $P_{\text {tr }}$ with different plate elasticity modulus, Figure 9(b) shows the spectra of power flow ratio PR = $10 \log \left(P_{\text {in }} / P_{\text {tr }}\right)$. The peaks of PR would always appear around the resonance frequencies except at $f_{\mathrm{C} 0}$, which could be easily explained by the fact that the plate's damping consumes more energy when system resonances take place. In the lowest frequency range around $f_{C 0}=0$, the plate consumes little energy, and therefore $P_{\mathrm{tr}} \approx P_{\text {in }}$ and $\mathrm{PR} \approx 0$. It should be noted that greater PRs might not imply lower levels of transmitted power flow $P_{\text {tr }}$; the fact is probably just the opposite because the power flow input $P_{\text {in }}$ might be in much higher levels at the same time. In this sense, minority of PR peak numbers would be a good design for noise isolation, which requires a greater plate elasticity modulus. And, instead, high PR values between adjacent resonance peaks of the PR spectra would be of real benefit for the purpose of $P_{\mathrm{tr}}$ attenuation, which could be discovered through a synthesized analysis of the figures shown.

In Figure 10(a), different mass density values were given to the elastic plate, in which the value of $2770 \mathrm{~kg} / \mathrm{m}^{3}$ is by reference to aluminium. A significant feature of the spectra in the figure is that changes in plate's mass density would apparently change the average level of transmitted power flow $P_{\text {tr }}$. According to (29), the increase of plate's mass density would cause decreasing of plate's natural frequencies and "frequency shifting" of fluid-structural coupled modals, as shown in Figure 10(a), similar to the situation of decreasing Young's modulus in Figure 9. However, the increase of plate's mass density would increase its modal masses at the same time. And that is the reason why $P_{\mathrm{tr}}$ 's average level is cut down even though more resonance modals would come into being in the relative lower frequency band. It could also be explained by the fact that a heavier vibrating mass would generate a greater reduction in dynamic force (or pressure) transmission. Figure $10(\mathrm{~b})$ is about the power flow ratio PR. PR could not effectively reveal the apparent $P_{\mathrm{tr}}$ reduction at nonresonance frequencies, such as $800 \mathrm{~Hz} \sim 1200 \mathrm{~Hz}$, because the transmitted power flow $P_{\mathrm{tr}}$ is very close to the power flow input $P_{\text {in }}$ at those frequencies.

Another important influential factor that should be paid attention is the damping loss factor of the plate. Figure 11(a) demonstrates a conflictive situation where smaller damping loss factor would increase the peaks of transmitted power flow at resonance frequencies, while at the broadband nonresonance frequencies smaller damping would be beneficial to reduction of transmitted power flow. To explain this result, one might make an analogy with the vibration isolation 


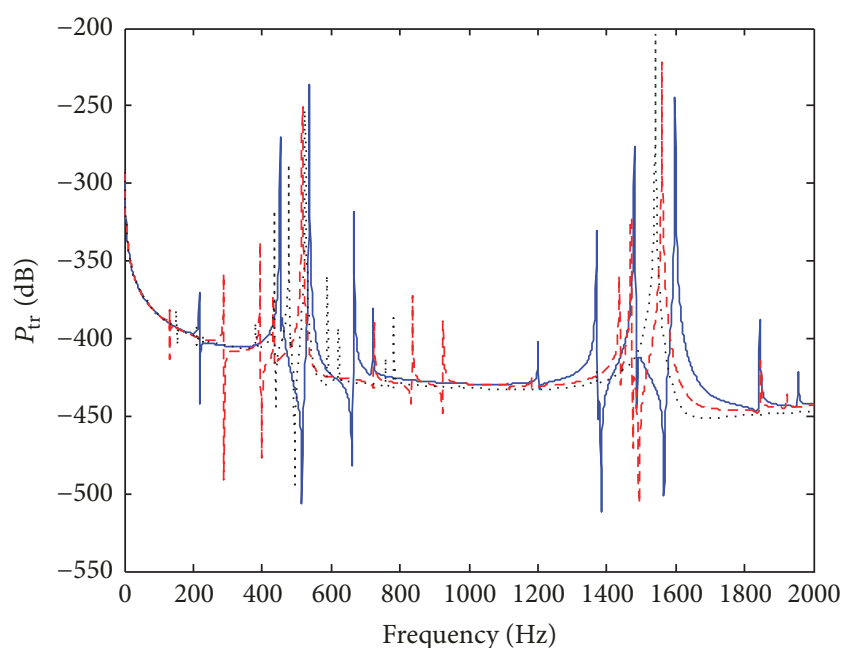

$$
\begin{aligned}
-E & =2.0 \times 10^{11} \mathrm{~Pa} \times\left(1+10^{-3} \mathrm{j}\right) \\
---E & =7.24 \times 10^{10} \mathrm{~Pa} \times\left(1+10^{-3} \mathrm{j}\right) \\
\cdots \cdots E & =2.0 \times 10^{10} \mathrm{~Pa} \times\left(1+10^{-3} \mathrm{j}\right)
\end{aligned}
$$

(a) Transmitted power flow

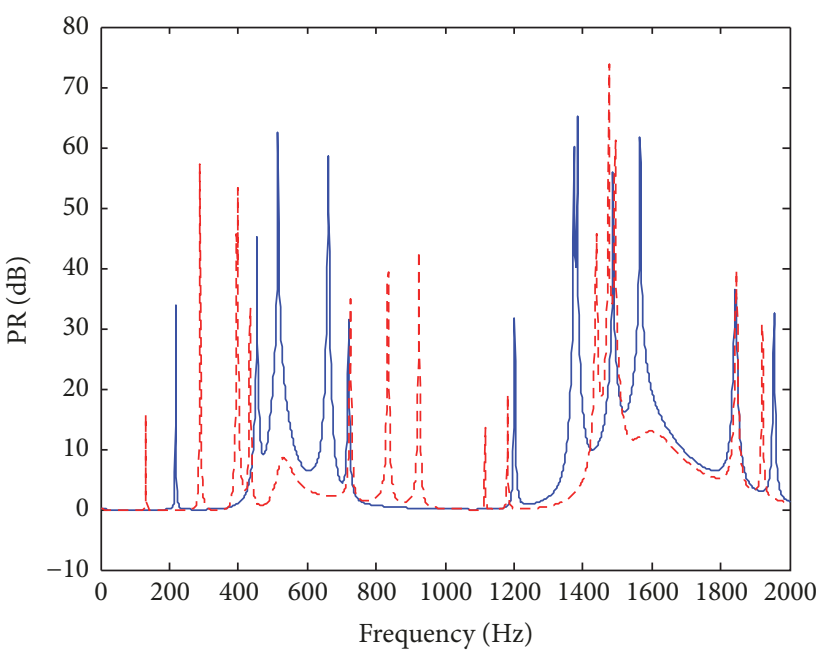

$-E=2.0 \times 10^{11} \mathrm{~Pa} \times\left(1+10^{-3} \mathrm{j}\right)$

$--E=7.24 \times 10^{10} \mathrm{~Pa} \times\left(1+10^{-3} \mathrm{j}\right)$

(b) Power flow ratio

FIGURE 9: Effect of plate's elasticity modulus on power flow transmission.

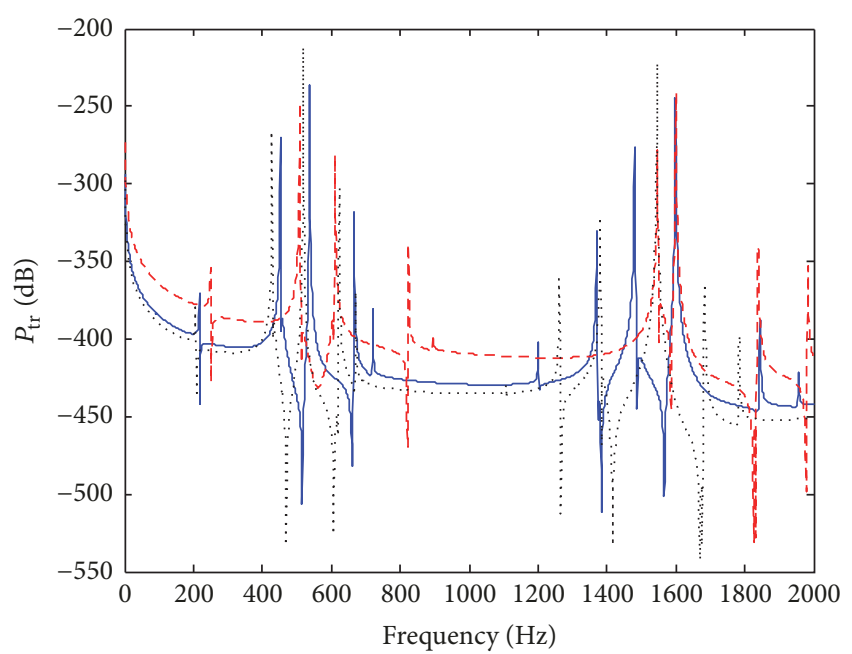

$\begin{aligned}-\rho & =7800 \mathrm{~kg} / \mathrm{m}^{3} \\ ---\rho & =2770 \mathrm{~kg} / \mathrm{m}^{3} \\ \ldots \ldots \rho & \rho=1 \times 10^{4} \mathrm{~kg} / \mathrm{m}^{3}\end{aligned}$

(a) Transmitted power flow

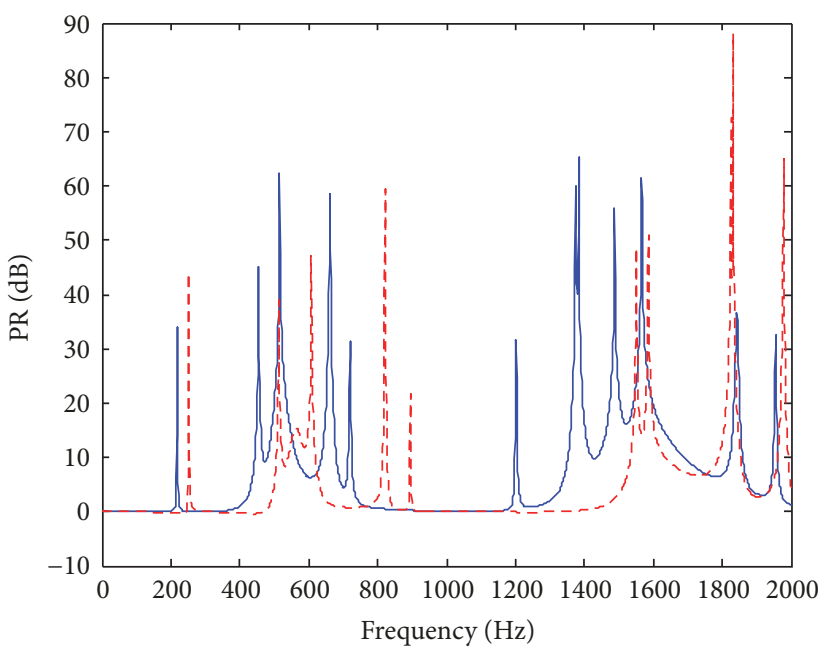

$\begin{aligned} \rho & =7800 \mathrm{~kg} / \mathrm{m}^{3} \\ & \end{aligned}$

(b) Power flow ratio

FIGURE 10: Effect of plate's mass density on power flow transmission.

theory. If the elastic plate was considered as some kind of elastic isolator which was specially designed to attenuate the transmission of exterior excitation energy into the waterfilled cavity, the damping would increase the power transmission and would not be expected except for attenuation of resonance peak. Through illustration of power flow ratio PR as that in Figure 11(b), it could be confirmed that greater damping could be used to obstruct energy transmission at resonance frequencies, whereas smaller damping would be favorable in the nonresonance frequency ranges, which would not be counted in power flow ratio.

Poisson's ratio $\mu$ would affect the power flow transmission the same way Young's modulus does as shown in Figure 9. Referring to (29), increasing Young's modulus could be equivalent to increasing Poisson's ratio. However, the variation scope of Poisson's ratio is much smaller than that of Young's modulus, and the power flow transmission would be affected more by Young's modulus than by Poisson's ratio. And, for this 


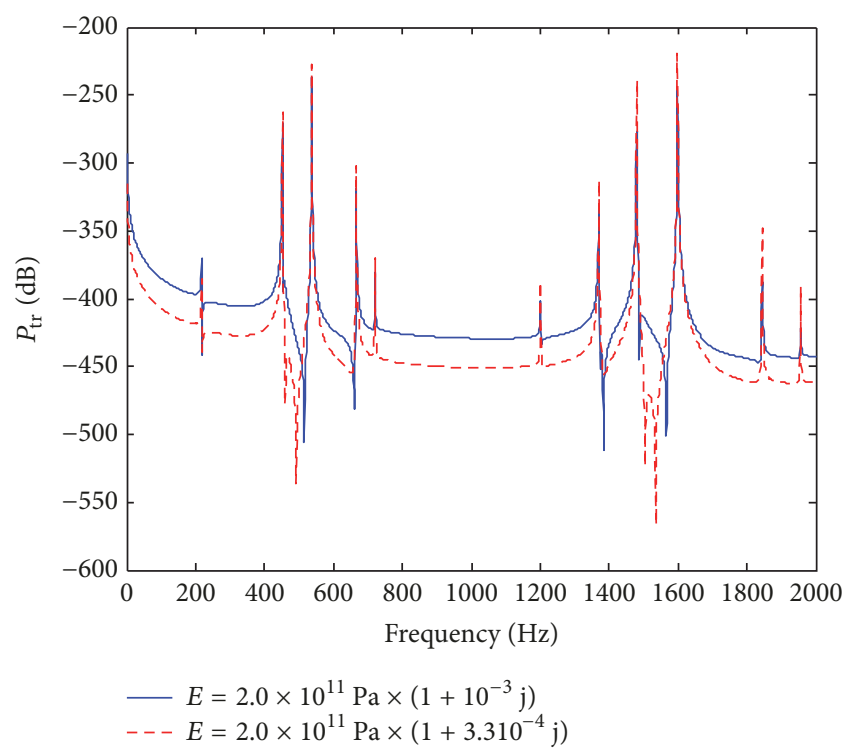

(a) Transmitted power flow

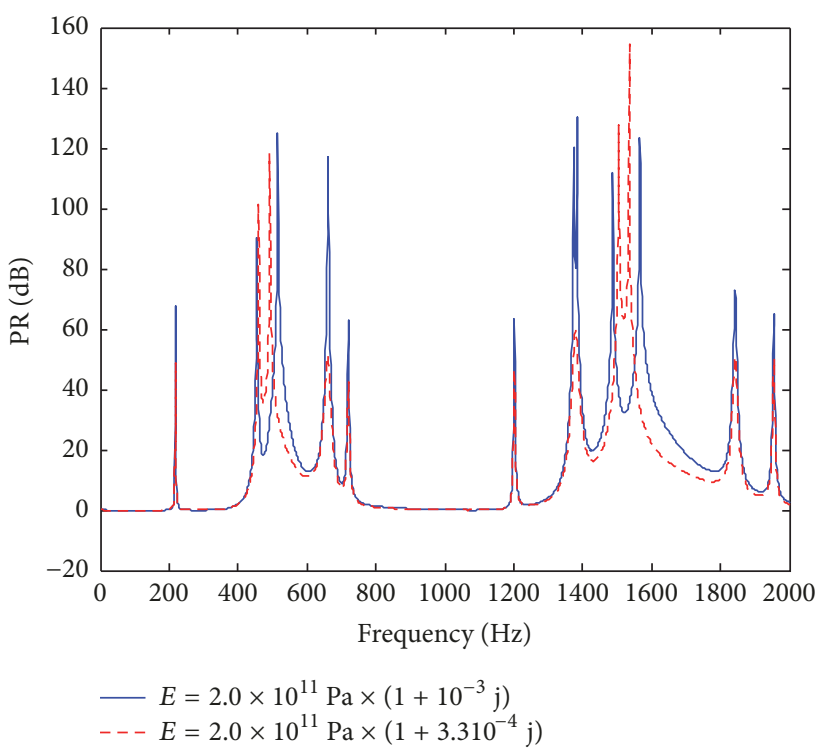

(b) Power flow ratio

FIGURE 11: Effect of damping loss factor of plate on power flow transmission.

reason, no additional repetitive figures would be put forward here.

\section{Conclusions}

Backgrounded by evaluation or control of mechanical selfnoise in sonar array cavity, transmitted power flow or sound power input calculation is carried out by modal coupling analysis on the fluid-structural vibration of the fluid-filled enclosure with elastic boundaries. Power flow transmission analysis is presented through a numerical simulation example of water-filled rectangular panel-cavity coupled system. Detailed discussion is carried out about power flow transmission characteristics affected by variation of material property parameters of cavity's elastic boundary structure, aiming at reduction of water sound level inside the cavity. From the results, one could draw the following conclusions.

(1) Power flow or sound power transmission analysis could be a valuable method for evaluation or prediction of water sound level in dealing with strong coupled vibration problems of water-filled acoustoelastic enclosure systems.

(2) The fluid-structural coupled natural frequencies of a water-filled acoustoelastic enclosure would be greatly affected by interaction between the hydroacoustic field and its surrounding elastic boundaries. There would be a tendency that the fluid-structural coupled natural frequencies turn to be smaller in value and more crowded in frequency distribution than those of elastic boundary structures and inner water sound field in rigid boundary condition.

(3) Decreasing elasticity modulus or Poison's ratio of water sound cavity's thin-wall structures would cause the frequency distribution of system modals to contract toward lower frequency ranges and result in more power flow transmission peaks in the lower frequency band, while slight reduction of peak valleys of transmitted power flow could also be expected on the other hand.

(4) Denser material could be beneficial to an apparent attenuation of average level of power flow transmission into the water-filled enclosure, even though it would be accompanied with a decrease of system natural frequencies.

(5) Smaller inner damping of enclosure's thin-wall structures, which were distinguished from that specially designed for sound absorption destination and only for the purpose of suppression of resonance peaks, might be proposed to attenuate the average level of power flow transmission.

\section{Appendix}

\section{A. Derivation Procedures of Related Matrices Equations}

A.1. Eigenvalue Problem. Equations (14) and (15) could be easily verified to be equivalent to the following simultaneous modal differential equations, which are fundamental in Dowell's modal coupling method:

$$
\begin{aligned}
& M_{A r} \ddot{P}_{r}+M_{A r} \omega_{A r}^{2} P_{r}=-\sum_{j=1}^{\infty} L_{r j} \ddot{B}_{j} \quad(r=0,1,2, \ldots) \\
& M_{B j} \ddot{B}_{j}+M_{B j} \omega_{B j}^{2} B_{j}=\sum_{r=0}^{\infty} L_{r j} P_{r}+Q_{B j} \\
&(j=1,2, \ldots),
\end{aligned}
$$

where $L_{r j}=\iint_{D_{F}} F_{A r} W_{B j} \mathrm{~d} A, Q_{B j}=-\iint_{D_{F}} W_{B j} p_{B} \mathrm{~d} A$, and all other symbols have been mentioned previously. 
Through some simple algebraic algorithm, (14) and (15) could be grouped together and written as

$$
\begin{aligned}
& {\left[\begin{array}{c}
\ddot{\mathbf{P}} \\
\ddot{\mathbf{B}}
\end{array}\right]+\left[\begin{array}{cc}
\boldsymbol{\Omega}_{A}^{2}+\mathbf{M}_{A}^{-1} \mathbf{L} \mathbf{M}_{B}^{-1} \mathbf{L}^{T} & -\mathbf{M}_{A}^{-1} \mathbf{L} \boldsymbol{\Omega}_{B}^{2} \\
-\mathbf{M}_{B}^{-1} \mathbf{L}^{T} & \boldsymbol{\Omega}_{B}^{2}
\end{array}\right]\left[\begin{array}{l}
\mathbf{P} \\
\mathbf{B}
\end{array}\right]} \\
& =\left[\begin{array}{c}
-\mathbf{M}_{A}^{-1} \mathbf{L} \mathbf{M}_{B}^{-1} \mathbf{Q}_{B} \\
\mathbf{M}_{B}^{-1} \mathbf{Q}_{B}
\end{array}\right] .
\end{aligned}
$$

While carrying out a modal analysis, one could simply let $\mathbf{P}=\chi_{A}^{\prime} \cdot \exp (j \omega t), \mathbf{B}=\boldsymbol{\chi}_{B}^{\prime} \cdot \exp (j \omega t)$, and $\mathbf{Q}_{B}=0$; thus

$$
\begin{aligned}
\omega^{2}\left[\begin{array}{l}
\chi_{A}^{\prime} \\
\chi_{B}^{\prime}
\end{array}\right] & =\left[\begin{array}{cc}
\boldsymbol{\Omega}_{A}^{2}+\mathbf{M}_{A}^{-1} \mathbf{L} \mathbf{M}_{B}^{-1} \mathbf{L}^{T} & -\mathbf{M}_{A}^{-1} \mathbf{L} \boldsymbol{\Omega}_{B}^{2} \\
-\mathbf{M}_{B}^{-1} \mathbf{L}^{T} & \boldsymbol{\Omega}_{B}^{2}
\end{array}\right]\left[\begin{array}{c}
\chi_{A}^{\prime} \\
\chi_{B}^{\prime}
\end{array}\right] \\
& =\mathbf{A}^{\prime}\left[\begin{array}{c}
\chi_{A}^{\prime} \\
\chi_{B}^{\prime}
\end{array}\right] .
\end{aligned}
$$

The above equation raises an eigenvalue problem, but it is nonstandard because the matrix $\mathbf{A}^{\prime}$ is asymmetric. The transformation expressed by (16) would result in a standard eigenvalue problem, which has been expressed by (17), where the matrix $\mathbf{A}$ is symmetric. And, moreover, one could prove that

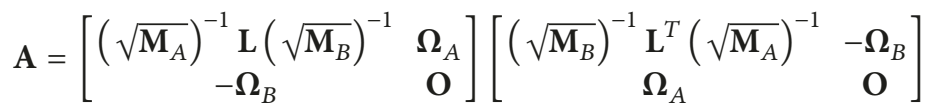

$$
\begin{aligned}
& =\left[\begin{array}{cc}
\left(\sqrt{\mathbf{M}_{A}}\right)^{-1} \mathbf{L}\left(\sqrt{\mathbf{M}_{B}}\right)^{-1} & \boldsymbol{\Omega}_{A} \\
-\boldsymbol{\Omega}_{B} & \mathbf{O}
\end{array}\right]\left[\begin{array}{cc}
\left(\sqrt{\mathbf{M}_{A}}\right)^{-1} \mathbf{L}\left(\sqrt{\mathbf{M}_{B}}\right)^{-1} & \boldsymbol{\Omega}_{A} \\
-\boldsymbol{\Omega}_{B} & \mathbf{O}
\end{array}\right]^{T},
\end{aligned}
$$

where $\mathbf{O}$ denotes a zero matrix with proper dimension rank.

Before any damping factors are introduced, (A.4) proves that the matrix $\mathbf{A}$ is positive semidefinite; that is, all its eigenvalues would be nonnegative. That would provide a mathematical guarantee that the eigenvalue calculation of matrix A would not fail at any occurrences of negative square natural frequencies.

By substituting any $k$ th eigenvalue of matrix $\mathbf{A}$ and its accompanying eigenvector, which have been symbolled with $\omega_{C k}{ }^{2}$ and $\left[\boldsymbol{\chi}^{(k)}\right]^{T}=\left[\left\{\boldsymbol{\chi}_{A}{ }^{(k)}\right\}^{T},\left\{\boldsymbol{\chi}_{B}{ }^{(k)}\right\}^{T}\right]$, respectively, into (16) (i.e., let $\omega=\omega_{C k}$ and $\chi_{A}=\chi_{A}{ }^{(k)}$ and $\left.\chi_{B}=\chi_{B}{ }^{(k)}\right)$, the $k$ th fluid-structural coupled modal of the cavity sound field and its flexible boundary structures, which have been symbolled with $F_{C k}$ and $W_{C k}$, respectively, are obtained by taking out the time-independent part of $\mathbf{P}(t)$ and $\mathbf{B}(t)$, that is, (18).

A.2. Harmonic Solution. In the situation of forced vibration, that is, $\mathbf{Q}_{B} \neq 0$, there is a standard decoupling procedure for (A.2) by applying an eigenmatrix $\mathbf{X}^{H}=\left[\mathbf{X}_{A}{ }^{H}, \mathbf{X}_{B}{ }^{H}\right]$ which is composed of the series of A's eigenvectors, where the detailed definitions of $\mathbf{X}_{A}$ and $\mathbf{X}_{B}$ have been mentioned previously in Section 2.2 after (19) and (20), and note that Hermitian transposition of matrices has been employed here in consideration of the introduction of damping loss factors. Replacing the real elasticity modulus of the flexible boundary structures or real volume stiffness of the cavity sound field with a complex elasticity modulus or complex volume stiffness is a regular method when damping factors are to be involved in analysis, which has been declared in Sections 2.2, 3.1, and 3.3; the method has also been adopted by many other authors.

Now let

$$
\left[\begin{array}{l}
\mathbf{P}(t) \\
\mathbf{B}(t)
\end{array}\right]=\left[\begin{array}{cc}
\left(\sqrt{\mathbf{M}_{A}}\right)^{-1} & \mathbf{O} \\
\mathbf{O} & \left(\mathbf{\Omega}_{B} \sqrt{\mathbf{M}_{B}}\right)^{-1}
\end{array}\right]\left[\begin{array}{l}
\mathbf{X}_{A} \\
\mathbf{X}_{B}
\end{array}\right] \boldsymbol{\alpha}(t)
$$

$$
=\mathbf{T}_{0} \cdot \mathbf{X} \cdot \boldsymbol{\alpha}(t),
$$

where $\boldsymbol{\alpha}(t)$ is a column vector variable to take place of $\mathbf{P}(t)$ and $\mathbf{B}(t)$.

After substituting the above equation into (A.2), multiply both sides of the resultant equation with a left-multiplication matrix $\mathbf{X}^{H} \mathbf{T}_{0}^{-1}$; one has

$$
\begin{gathered}
\mathbf{X}^{H} \mathbf{T}_{0}^{-1} \mathbf{T}_{0} \mathbf{X} \cdot \ddot{\boldsymbol{\alpha}}+\mathbf{X}^{H} \mathbf{T}_{0}^{-1} \mathbf{A}^{\prime} \mathbf{T}_{0} \mathbf{X} \cdot \boldsymbol{\alpha} \\
=\mathbf{X}^{H_{1}} \mathbf{T}_{0}^{-1}\left[\begin{array}{c}
-\mathbf{M}_{A}^{-1} \mathbf{L} \mathbf{M}_{B}^{-1} \mathbf{Q}_{B} \\
\mathbf{M}_{B}^{-1} \mathbf{Q}_{B}
\end{array}\right]
\end{gathered}
$$

Take notice that $\mathbf{T}_{0}^{-1} \mathbf{A}^{\prime} \mathbf{T}_{0}=\mathbf{A}$, and $\mathbf{X}^{H} \mathbf{X}$ and $\mathbf{X}^{H} \mathbf{A X}$ are all diagonal matrices according to the theory of orthogonality of eigenvectors. In fact, the diagonal elements of matrix $\mathbf{M}_{C}=\mathbf{X}^{H} \mathbf{X}$ are just the modal masses of the fluid-structural coupled modals, and the diagonal elements of matrix $\Omega_{C}^{2}=$ $\mathbf{M}_{C}^{-1}\left(\mathbf{X}^{H} \mathbf{A X}\right)$ are the complex fluid-structural coupled natural frequencies; these two matrices have already been defined in Section 2.2 after (20). With this knowledge, (A.2) or (A.6) is turned into its decoupled expression:

$$
\ddot{\boldsymbol{\alpha}}+\boldsymbol{\Omega}_{C}^{2} \boldsymbol{\alpha}=\mathbf{M}_{C}^{-1} \mathbf{X}^{H} \mathbf{T}_{0}^{-1}\left[\begin{array}{c}
-\mathbf{M}_{A}^{-1} \mathbf{L M}_{B}^{-1} \mathbf{Q}_{B} \\
\mathbf{M}_{B}^{-1} \mathbf{Q}_{B}
\end{array}\right] .
$$

Obviously, the transient or steady solution of the above equation under arbitrary deterministic $\mathbf{Q}_{B}(t)$ could be obtained by the method of convolution integral. Only harmonic solution is concerned here. Referring to (12), when $p_{B}(\sigma, t)$ is harmonic, $\mathbf{Q}_{B}(t)$ is harmonic, too. And suppose that $\mathbf{Q}_{B}(t)=\mathbf{P}_{B} \cdot \exp (j \omega t)$ and $\boldsymbol{\alpha}(t)=\overline{\boldsymbol{\alpha}} \exp (j \omega t)$; by substituting them into (A.7), the time-dependent part $\exp (j \omega t)$ 
would be eliminated, and the time-independent part of $\boldsymbol{\alpha}(t)$ is obtained; that is,

$$
\begin{aligned}
& \overline{\boldsymbol{\alpha}}=\left(-\omega^{2} \mathbf{I}+\mathbf{\Omega}_{C}^{2}\right)^{-1} \mathbf{M}_{C}^{-1} \mathbf{X}^{H} \mathbf{T}_{0}^{-1}\left[\begin{array}{c}
-\mathbf{M}_{A}^{-1} \mathbf{L M}_{B}^{-1} \mathbf{P}_{B} \\
\mathbf{M}_{B}^{-1} \mathbf{P}_{B}
\end{array}\right] \\
& =\left(-\omega^{2} \mathbf{M}_{C}+\mathbf{\Omega}_{C}^{2} \mathbf{M}_{C}\right)^{-1} \\
& \cdot\left[\begin{array}{ll}
\mathbf{X}_{A}^{H} & \mathbf{X}_{B}^{H}
\end{array}\right]\left[\begin{array}{cc}
\sqrt{\mathbf{M}_{A}} & \mathbf{O} \\
\mathbf{O} & \mathbf{\Omega}_{B} \sqrt{\mathbf{M}_{B}}
\end{array}\right]\left[\begin{array}{c}
-\mathbf{M}_{A}^{-1} \mathbf{L M}_{B}^{-1} \mathbf{P}_{B} \\
\mathbf{M}_{B}^{-1} \mathbf{P}_{B}
\end{array}\right] \\
& =\mathbf{H}(\omega) \\
& \cdot\left[-\mathbf{X}_{A}^{H}\left(\sqrt{\mathbf{M}_{A}}\right)^{-1} \mathbf{L M}_{B}^{-1}+\mathbf{X}_{B}^{H} \boldsymbol{\Omega}_{B}\left(\sqrt{\mathbf{M}_{B}}\right)^{-1}\right] \mathbf{P}_{B} \\
& =\mathbf{H}(\omega) \cdot \mathbf{T} \cdot \mathbf{P}_{B},
\end{aligned}
$$

where $\mathbf{I}$ is a unit matrix of proper dimension rank.

A.3. Power Flow Formulation. The basic formulation for power flow computation is

$$
\begin{aligned}
P_{F V}= & \frac{\omega}{2 \pi} \int_{0}^{2 \pi / \omega} F \cos \omega t \cdot V \cos (\omega t+\varphi) \mathrm{d} t \\
= & \frac{\omega}{2 \pi} \int_{0}^{2 \pi / \omega} \operatorname{Re}\{F \exp (j \omega t)\} \\
& \cdot \operatorname{Re}\{V \exp (j \omega t+j \varphi)\} \mathrm{d} t=\frac{1}{2} F V \cos \varphi \\
= & \frac{1}{2} \operatorname{Re}\left\{[F \exp (j \omega t)]^{*}[V \exp (j \omega t+j \varphi)]\right\} \\
= & \frac{1}{2} \operatorname{Re}\left\{[F \exp (j \omega t)][V \exp (j \omega t+j \varphi)]^{*}\right\},
\end{aligned}
$$

where $F \cos \omega t$ or $F \exp (j \omega t)$ is a harmonic excitation force, $V \cos \omega t$ or $V \exp (j \omega t+j \varphi)$ is the harmonic velocity response at the action point of the excitation force, and $\varphi$ is the phase difference between the excitation force and velocity response.

By applying the above formulation, (23) would be apparent. And because $P_{B}(\sigma)$ is of $\sigma$-dependent distribution over $D_{F}, p_{\text {in }}(\sigma, \omega)$ in (23) is of distribution of power flow density. Equation (25) is an integral of $p_{\text {in }}$ over $D_{F}$ to count up the total power flow. The integral is initiated by quotation of (22) and (23) as follows:

$$
\begin{aligned}
& P_{\text {in }}(\omega)=-\iint_{D_{F}} \frac{\omega}{2} \operatorname{Re}\left\{j W(\sigma, \omega) \cdot P_{B}(\sigma)\right\} \mathrm{d} A \\
& =-\iint_{D_{F}} \frac{\omega}{2} \operatorname{Re}\left\{j[W(\sigma, \omega)]^{T} \cdot P_{B}^{*}(\sigma)\right\} \mathrm{d} A
\end{aligned}
$$

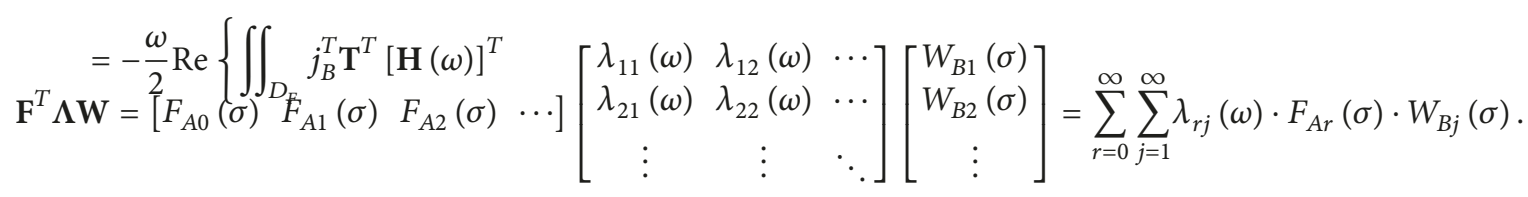

Note that in the last expression of the right hand of the above equation, the matrices $\mathbf{P}_{B}, \mathbf{T}, \mathbf{H}(\omega), \mathbf{X}_{B}, \boldsymbol{\Omega}_{B}$, and $\mathbf{M}_{B}$ are all $\sigma$-independent; only $\mathbf{W}(\sigma) \cdot P_{B}^{*}(\sigma)$ would join in the integral in (A.10). The integral would result in $\left(\mathbf{P}_{B}\right)^{*}$, in which $\mathbf{P}_{B}$ has been defined in the beginning paragraph of Section 2.3, and the superscript “ $*$ " denotes conjugation of complex numbers. Other matters need attention; $\mathbf{H}(\omega), \boldsymbol{\Omega}_{B}$, and $\mathbf{M}_{B}$ are all diagonal matrices; that is, $[\mathbf{H}(\omega)]^{T}=\mathbf{H}(\omega)$ and $\left[\left(\boldsymbol{\Omega}_{B} \sqrt{\mathbf{M}_{B}}\right)^{-1}\right]^{T}=\left(\boldsymbol{\Omega}_{B} \sqrt{\mathbf{M}_{B}}\right)^{-1}$. After taking into account all above factors, (24) is realized.

The obtainment of (25) is similar to that of (23), in which the cavity sound pressure at the interior surface of the thinwall structures takes place of the exterior excitation. And also the integral of $p_{\operatorname{tr}}(\sigma, \omega)$ is processed similar to that of $p_{\text {in }}(\sigma, \omega)$ at the beginning by quotation of (22) and (25).

$$
\begin{aligned}
& P_{\mathrm{tr}}(\omega)=\iint_{D_{F}} \frac{\omega}{2} \operatorname{Re}\left\{\left.j W^{*}(\sigma, \omega) \cdot P(\sigma, \omega)\right|_{\sigma \in D_{F}}\right\} \mathrm{d} A \\
& =\iint_{D_{F}} \frac{\omega}{2} \operatorname{Re}\{j P(\sigma, \omega) \\
& \left.\left.\cdot[W(\sigma, \omega)]^{H}\right|_{\sigma \in D_{F}}\right\} \mathrm{d} A=\frac{\omega}{2} \\
& \cdot \operatorname{Re}\left\{\iint_{D_{F}} j[\mathbf{F}(\sigma)]^{T}\left(\sqrt{\mathbf{M}_{A}}\right)^{-1}\right. \\
& \cdot \mathbf{X}_{A} \mathbf{H T P}_{B} \mathbf{P}_{B}^{H} \mathbf{T}^{H} \mathbf{H}^{H} \mathbf{X}_{B}^{H}\left[\left(\boldsymbol{\Omega}_{B} \sqrt{\mathbf{M}_{B}}\right)^{-1}\right]^{H} \\
& \cdot \mathbf{W}(\sigma) \mathrm{d} A\}=\frac{\omega}{2} \operatorname{Re}\left\{\iint_{D_{F}}[\mathbf{F}(\sigma)]^{T}\right. \\
& {\left[j\left(\sqrt{\mathbf{M}_{A}}\right)^{-1}\right.} \\
& \left.\cdot \mathbf{X}_{A} \mathbf{H T P}_{B} \mathbf{P}_{B}^{H} \mathbf{T}^{H} \mathbf{H}^{*} \mathbf{X}_{B}^{H}\left(\boldsymbol{\Omega}_{B}^{*} \sqrt{\mathbf{M}_{B}}\right)^{-1}\right] \\
& \cdot \mathbf{W}(\sigma) \mathrm{d} A\} \text {. }
\end{aligned}
$$

The basis of the above transformation includes the following: $\mathbf{W}(\sigma)$ is real, $\mathbf{H}$ and $\boldsymbol{\Omega}_{B}$ are diagonal, and $\mathbf{M}_{B}$ is real and diagonal. It could be examined that the product of the 
TABLE 4: Dimension ranks of related matrices with $R$ cavity acoustical modals and $N$ thin-wall structural modals.

\begin{tabular}{|c|c|c|c|c|c|}
\hline Matrices & Dimension rank & Matrices & Dimension rank & Matrices & Dimension rank \\
\hline$\nabla F_{A r}$ & $3 \times 1$ & F & $R \times 1$ & $\mathbf{P}$ & $R \times 1$ \\
\hline$\nabla \mathbf{F}$ & $3 \times R$ & $\mathbf{M}_{A}$ & $R \times R$ & $\boldsymbol{\Omega}_{A}$ & $R \times R$ \\
\hline W & $N \times 1$ & B & $N \times 1$ & $\mathbf{M}_{B}$ & $N \times N$ \\
\hline $\boldsymbol{\Omega}_{B}$ & $N \times N$ & $\mathbf{Q}_{A}$ & $N \times 1$ & $\mathbf{Q}_{B}$ & $N \times 1$ \\
\hline $\mathbf{L}$ & $R \times N$ & $\chi_{A}, \boldsymbol{\chi}_{A}{ }^{(k)}, \boldsymbol{\chi}_{A}^{\prime}$ & $R \times 1$ & $\boldsymbol{\chi}_{B}, \boldsymbol{\chi}_{B}^{(k)}, \boldsymbol{\chi}_{B}^{\prime}$ & $N \times 1$ \\
\hline$\chi, \chi^{(k)}$ & $(R+N) \times 1$ & $\mathbf{A}, \mathbf{A}^{\prime}$ & $(R+N) \times(R+N)$ & $\mathbf{A}_{11}$ & $R \times R$ \\
\hline $\mathbf{A}_{12}$ & $R \times N$ & $\mathbf{A}_{21}$ & $N \times R$ & $\mathbf{A}_{22}$ & $N \times N$ \\
\hline $\mathbf{X}_{A}$ & $R \times(R+N)$ & $\mathbf{X}_{B}$ & $N \times(R+N)$ & $\mathbf{X}$ & $(R+N) \times(R+N)$ \\
\hline$S$ & $(R+N) \times 1$ & $\mathbf{H}$ & $(R+N) \times(R+N)$ & $\mathrm{T}$ & $(R+N) \times N$ \\
\hline$S_{Q}$ & $N \times 1$ & $\mathbf{M}_{C}$ & $(R+N) \times(R+N)$ & $\boldsymbol{\Omega}_{C}$ & $(R+N) \times(R+N)$ \\
\hline $\mathbf{P}_{B}$ & $N \times 1$ & $\Lambda$ & $R \times N$ & $\mathrm{~T}_{0}$ & $(R+N) \times(R+N)$ \\
\hline$\alpha, \bar{\alpha}$ & $(R+N) \times 1$ & & & & \\
\hline
\end{tabular}

Since $\lambda_{r j}(\omega)$ is $\sigma$-independent, when the integral of $[\mathbf{F}(\sigma)]^{T} \boldsymbol{\Lambda}(\omega) \mathbf{W}(\sigma)$ over $D_{F}$ is proceeding, only $F_{A r}(\sigma) W_{B j}(\sigma)$ is involved, and that would result in $L_{r j}$, which has been defined in (13) or (A.1). And, at last, $P_{\mathrm{tr}}(\omega)$ is formulated by (26).

\section{B. Dimension Ranks of Related Matrices under Modal Truncation}

Modal truncation has to be implemented in the application of modal coupling method, because there are no numerical techniques that could provide a computation in infinity manner by now. And because of the modal convergence property, modal truncation errors could be controlled properly. Suppose that there are totally $R$ cavity acoustical modals and $N$ thin-wall structural modals to be accounted for the fluidstructural modal coupling; dimension ranks of associated matrices mentioned in this paper are listed in Table 4 .

\section{Conflicts of Interest}

The authors declare that there are no conflicts of interest regarding the publication of this paper.

\section{References}

[1] H. Guo, D. Luo, M. Chen, and F. Zhou, "Prediction method for low frequency of self-noise in submarine's fore body sonar platform area," Ship Science and Technology, vol. 27, no. 4, pp. 74-77, 2005.

[2] M. Yu, J. Ye, Y. Wu, and S. Lu, "Prediction and control method of self-noise in ship's sonar domes," Journal of Ship Mechanics, vol. 6, no. 5, pp. 80-94, 2002.

[3] T. Musha and T. Kikuchi, "Numerical calculation for determining sonar self noise sources due to structural vibration," Applied Acoustics, vol. 58, no. 1, pp. 19-32, 1999.

[4] S. Narayanan and R. L. Shanbhag, "Sound transmission through elastically supported sandwich panels into a rectangular enclosure," Journal of Sound and Vibration, vol. 77, no. 2, pp. 251-270, 1981.
[5] G. B. Warburton, "Vibration of a cylindrical shell in an acoustic medium," Journal of Mechanical Engineering Science, vol. 3, no. 1, pp. 69-79, 1961.

[6] J.-M. David and M. Menelle, "Validation of a medium-frequency computational method for the coupling between a plate and a water-filled cavity," Journal of Sound and Vibration, vol. 265, no. 4, pp. 841-861, 2003.

[7] J. Yang, X. Li, and J. Tian, "Theoretical analysis of sound transmission through viscoelastic sandwich panel backed by a cavity," ACTA Acustica, vol. 24, no. 6, pp. 617-626, 1999.

[8] J. Yang, Y. P. Xiong, and J. T. Xing, "Vibration power flow and force transmission behaviour of a nonlinear isolator mounted on a nonlinear base," International Journal of Mechanical Sciences, vol. 115-116, pp. 238-252, 2016.

[9] P. M. Morse, "Some aspects of the theory of room acoustics," The Journal of the Acoustical Society of America, vol. 11, no. 1, pp. 56-66, 1939.

[10] J. Pan, "The forced response of an acoustic-structural coupled system," The Journal of the Acoustical Society of America, vol. 91, no. 2, pp. 949-956, 1992.

[11] S. R. Bistafa and J. W. Morrissey, "Numerical solutions of the acoustic eigenvalue equation in the rectangular room with arbitrary (uniform) wall impedances," Journal of Sound and Vibration, vol. 263, no. 1, pp. 205-218, 2003.

[12] E. H. Dowell, G. F. Gorman III, and D. A. Smith, "Acoustoelasticity: General theory, acoustic natural modes and forced response to sinusoidal excitation, including comparisons with experiment," Journal of Sound and Vibration, vol. 52, no. 4, pp. 519-542, 1977.

[13] E. H. Dowell, "Comment on 'On Dowell's simplification for acoustic cavity-structure interaction and consistent alternatives," The Journal of the Acoustical Society of America, vol. 133, no. 3, pp. 1222-1224, 2013.

[14] R. W. Guy, "The response of a cavity backed panel to external airborne excitation: A general analysis," The Journal of the Acoustical Society of America, vol. 65, no. 3, pp. 719-731, 1979.

[15] J. H. Ginsberg, "Derivation of a Ritz series modeling technique for acoustic cavity-structural systems based on a constrained Hamiltons principle," The Journal of the Acoustical Society of America, vol. 127, no. 5, pp. 2749-2758, 2010. 
[16] V. B. Bokil and U. S. Shirahatti, "A technique for the modal analysis of sound-structure interaction problems," Journal of Sound and Vibration, vol. 173, no. 1, pp. 23-41, 1994.

[17] N. Kang and A. Raman, "Aeroelastic flutter mechanisms of a flexible disk rotating in an enclosed compressible fluid," Journal of Applied Mechanics, vol. 71, no. 1, pp. 120-130, 2004.

[18] J. Pan and D. A. Bies, "The effect of fluid-structural coupling on sound waves in an enclosure-theoretical part," The Journal of the Acoustical Society of America, vol. 87, no. 2, pp. 691-707, 1990.

[19] J. Pan and D. A. Bies, "The effect of fluid-structural coupling on sound waves in an enclosure-experimental part," The Journal of the Acoustical Society of America, vol. 87, no. 2, pp. 708-717, 1990.

[20] R. B. Davis, "A simplified approach for predicting interaction between flexible structures and acoustic enclosures," Journal of Fluids and Structures, vol. 70, pp. 276-294, 2017. 


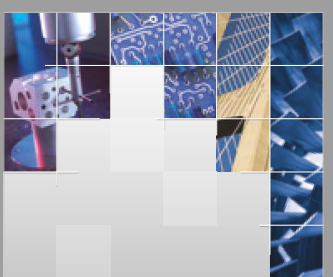

\section{Enfincering}
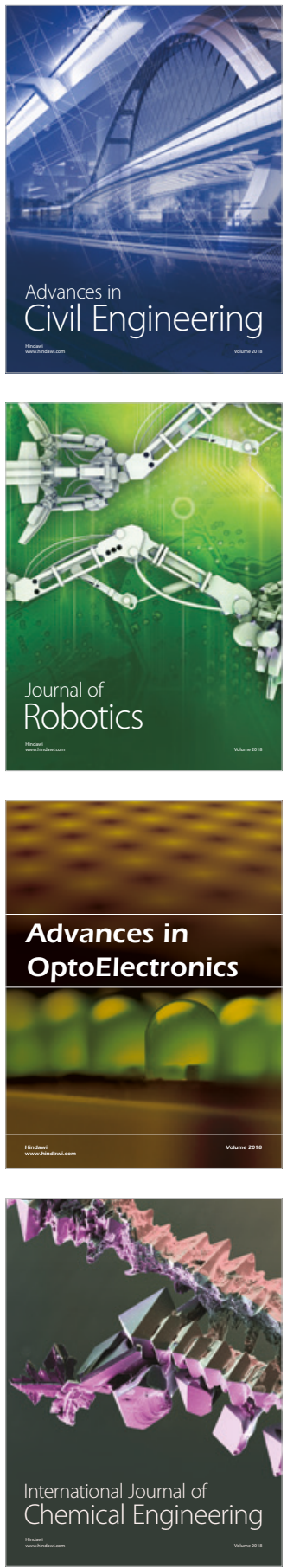

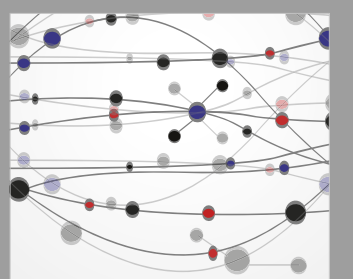

\section{Rotating \\ Machinery}

The Scientific World Journal

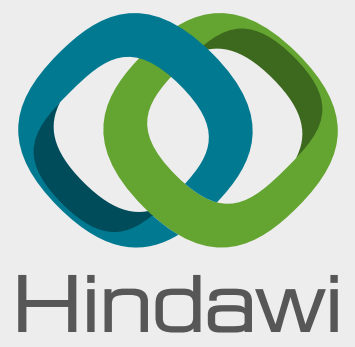

Submit your manuscripts at

www.hindawi.com
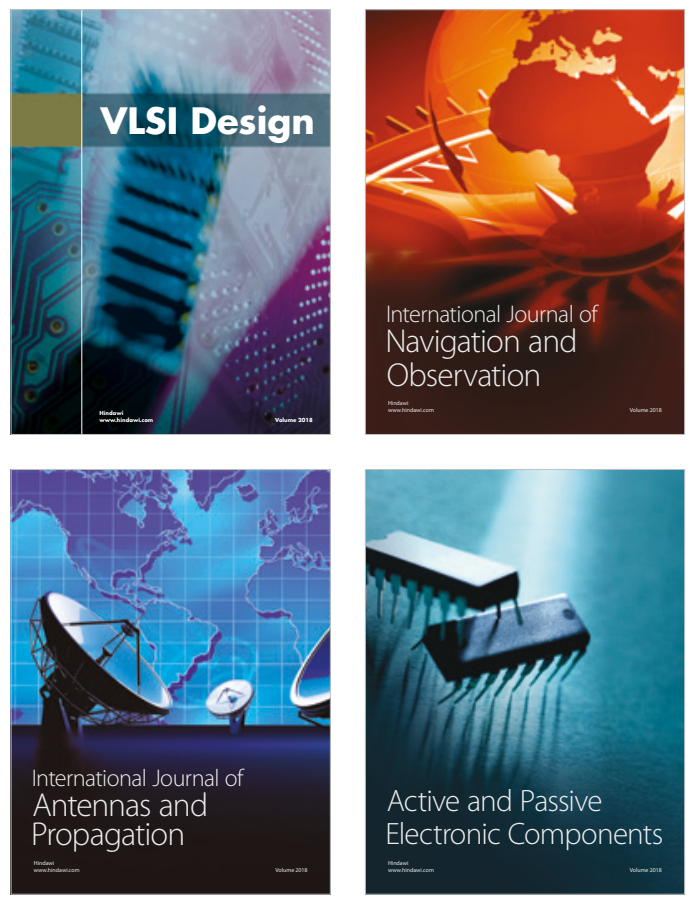
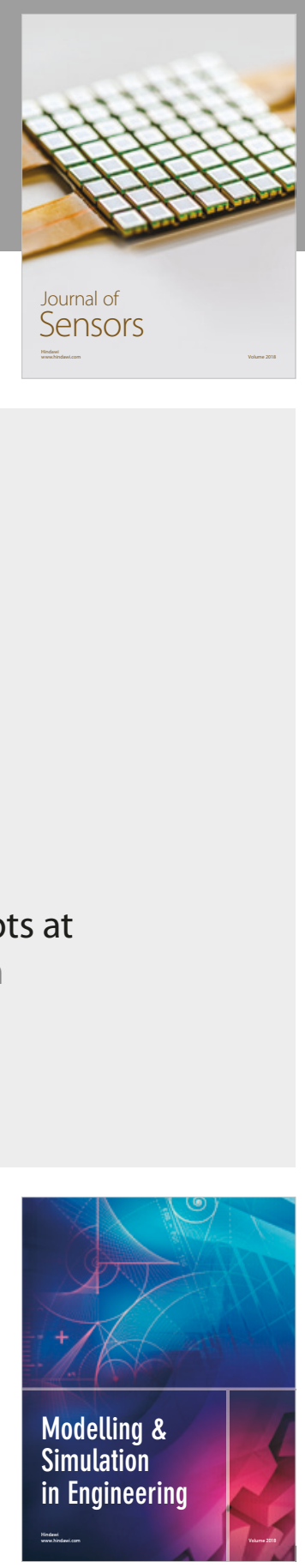

\section{Advances \\ Multimedia}
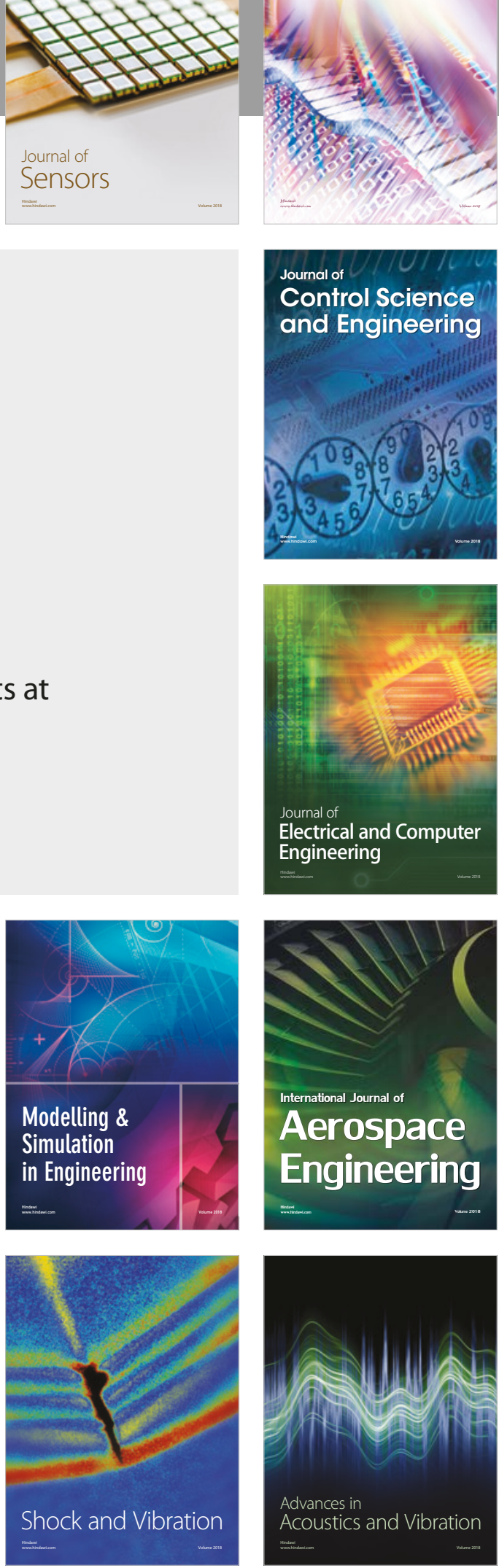